\title{
Natural-Color D-to-Z Diamonds: A Crystal-Clear Perspective
}

Sally Eaton-Magaña, Troy Ardon, Christopher M. Breeding, and James E. Shigley

Colorless to light yellow or brown diamonds with a "D-to-Z" color grade make up the overwhelming majority of the world's gem diamond trade. Besides clarity features (such as inclusions) and fluorescence observations, however, comparatively little has been explored and published regarding the distinguishing characteristics of these diamonds. The vast majority are type la, with infrared spectra showing very high concentrations of nitrogen aggregates. This population of diamonds could not have been subjected to HPHT decolorizing treatment or been laboratory grown, and thus they have been spectroscopically scrutinized in much less detail than the far more rare natural diamonds of types Ila, IIb, and IaB, which need to be investigated as potentially color-treated or synthetic.

This study examines a large sample set comprising the full complement of D-to-Z diamonds submitted to GIA laboratories during a significant portion of 2017. The data were evaluated on the basis of diamond type properties, as well as distribution among various grading quality factors, to provide an unprecedented glimpse into the role of these diamond types and differences in their geologic conditions of formation.

W hen people hear the word diamond, colorless diamonds generally come to mindnot the more exotic colors discussed earlier in this article series. In purchasing a diamond, they are likely to choose one that is colorless to nearcolorless. When one thinks of perfection in a diamond, a D-Flawless stone is usually the benchmark. The lack of color, which so inhibits the consumer appeal of many other gemstones, is instead considered a measure of stature for diamonds.

Therefore, we finish this series (Breeding et al., 2018, 2020; Eaton-Magaña et al., 2018a, 2018b, 2019) by examining diamonds with very little to no color at all-those on the D-to-Z grading scale. Although they make up by far the largest proportion of stones submitted to GIA, detailed statistics of D-to-Z diamonds have never been presented before. In each of our previous colored diamond articles, we presented data on stones submitted to GIA from 2008 to 2016. For example, GIA examined more than 15,000 naturally colored blue/gray/violet diamonds in that period (Eaton-Magaña et al., 2018a). During those same years, GIA received millions of $\mathrm{D}$-to-Z diamonds (e.g., figure 1).

See end of article for About the Authors and Acknowledgments.

Gems \& Gemology, Vol. 56, No. 3, pp. 318-335,

http://dx.doi.org/10.5741/GEMS.56.3.335

(C) 2020 Gemological Institute of America
For this study, we examined all D-to-Z diamonds submitted for grading reports or dossier reports to any of GIA's laboratories during much of 2017. From our analysis of submissions over the last decade, those from 2017 and used in this study are representative of GIA's current and historical intake. Any diamonds submitted on multiple occasions during this period were identified, and only the most recent data for each stone are included in this study.

\section{In Brief}

- D-Z diamonds represent the vast majority of all gem diamonds but have rarely been documented systematically and in detail from a gemological laboratory.

- When D-Z diamonds are distinguished by diamond type, we see distinct trends in their grading quality factors.

- HPHT-treated diamonds have distinct trends in their grading quality factors compared to their natural counterparts.

Additionally, we wish to highlight that these data represent a snapshot for a certain period and only include those diamonds submitted to GIA. They may not represent the entirety of the marketplace, such as some lower-quality diamonds that are not 


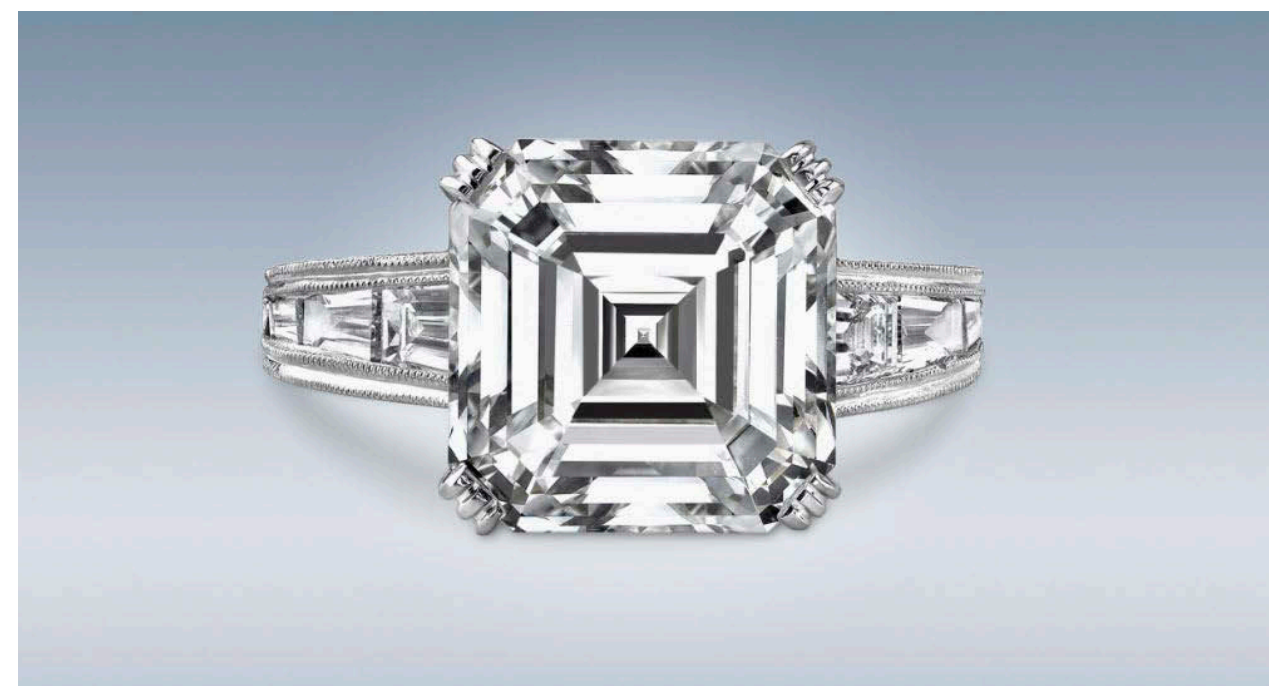

Figure 1. Diamond rings that commemorate a marriage engagement often utilize colorless to near-colorless diamonds and constitute a major sector of the gem and jewelry world. Photo courtesy of Neil Lane.

usually submitted for grading. However, for those in the marketplace that are certified, these results are representative.

The trends presented in this article apply to faceted diamonds. They represent not only the rough selected for manufacturing but also the business and cutting decisions of the polishers. Each faceted diamond is ultimately the culmination and intersection of science, artistry, and commerce.

The GIA system for grading (the absence of) color with the D-to-Z scale has gained worldwide trade and consumer acceptance since its introduction in 1953 by Richard T. Liddicoat and Robert Crowningshield, followed by the first diamond grading reports issued by GIA in 1955 (Dirlam et al., 2002). The grading terminology (though not necessarily the practices) has since been adopted by most other gemological laboratories (King et al., 2008).

Many articles about various aspects of diamond grading have been published regarding color grading (King et al., 2006, 2008), cutting decisions (Caspi, 1997), cut grading of round brilliants (Hemphill et al., 1998; Moses et al., 2004), symmetry (Geurts et al., 2011), crystal inclusions (Koivula, 2000; Kaminsky et al., 2000; Shirey and Shigley, 2013; Smith et al., 2016; Renfro et al., 2018), trends exclusively seen in large diamonds (Smith et al., 2017), and fluorescence (Moses et al., 1997; Luo and Breeding, 2013; Bouman et al., 2018; Breeding and Eaton-Magaña, 2019). Recounting the specifics of the grading process or the mechanics of fluorescence observations is beyond the scope of this article. Instead, we present some statistics of these grading quality factors and other recorded data to illuminate relationships and trends not recognized before.

\section{CAUSES OF COLOR}

Only diamonds deemed to be colorless or possessing faint to light yellow or brown color are graded according to the D-to-Z color scale (figure 2). Diamonds with faint to light color saturation of other hues are considered "fancy color" (King et al., 2008), and many of those categories have been described previously in this series. The causes of yellow coloration for D-to-Z diamonds are similar to those for Fancy yellow color (Breeding et al., 2020) and brown coloration (Eaton-Magaña et al., 2018b), but these color causes will be briefly recounted here.

Stones with grayish coloration were discussed in detail in Eaton-Magaña et al. (2018a). In most natural diamonds, the grayish color is produced by clouds of dark (often graphitic) inclusions. For grayish diamonds with a depth of color equivalent to K or greater, the diamond is given a color description of Faint gray, Very Light gray, etc., but without a letter grade (King et al., 2008). Therefore, only D to J diamonds (that is, colorless to near-colorless) with a grayish color contribution would be placed on the D-to-Z scale.

Yellow. The majority $(\sim 74 \%)$ of Fancy yellow diamonds submitted to GIA are colored by "cape" spectral features (figure 3), which include a combination of absorptions from the N3 defect (three nitrogen atoms adjacent to a vacancy and a zero-phonon line at $415.2 \mathrm{~nm}$ ), the associated $\mathrm{N} 2$ band at $478 \mathrm{~nm}$, and absorptions at 451 and $463 \mathrm{~nm}$ (Breeding et al., 2020). The term cape, originally used for yellow diamonds from Cape Province in South Africa (the first prominent source of such diamonds), is now used for any yellow diamond colored by N3 and its associated defects. 


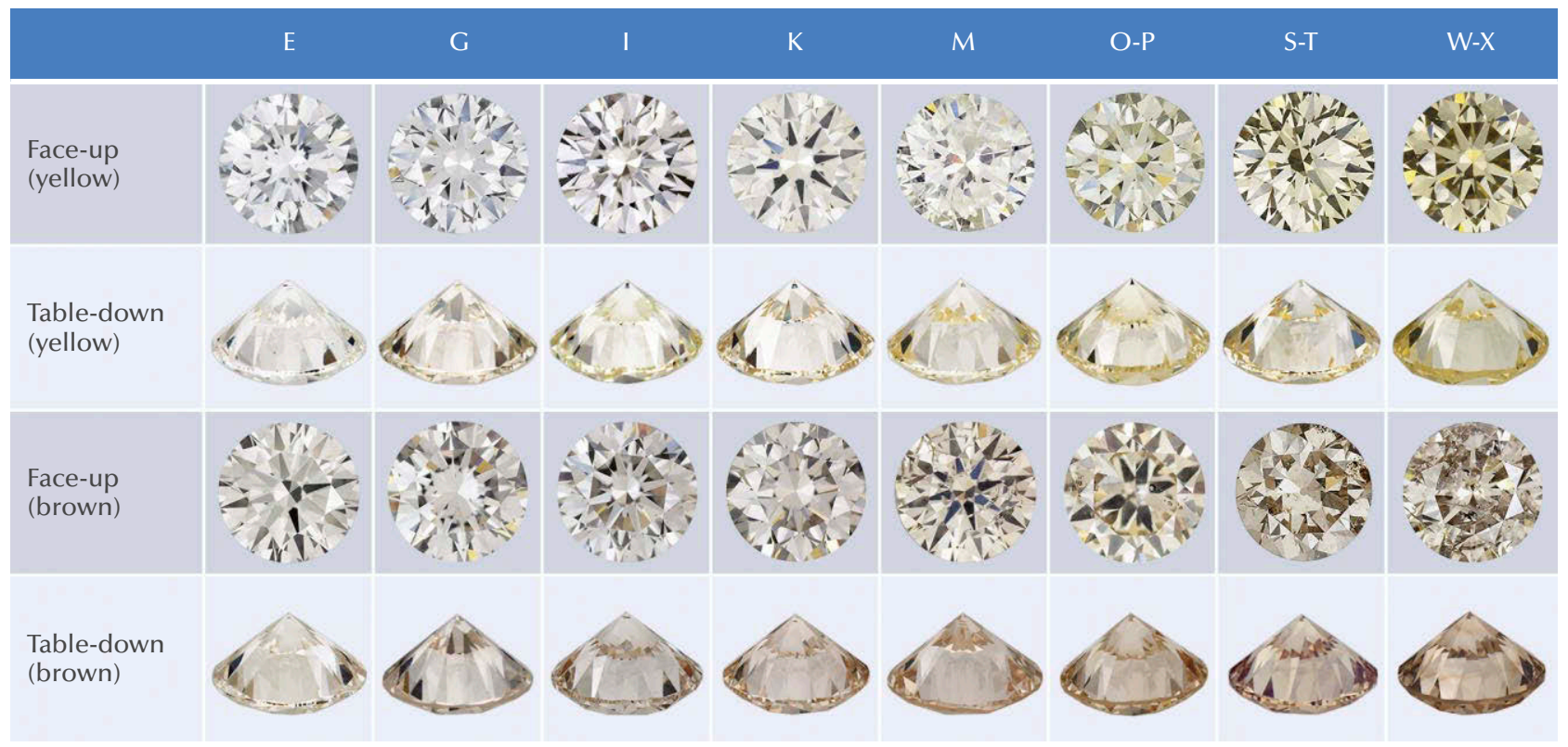

Figure 2. The color grades of $D$-to- $Z$ diamonds are generally assessed in the table-down position (King et al., 2008) but admired in jewelry in face-up orientation. While most $D$-to- $Z$ diamonds show a yellow coloration, a small percentage (about $5 \%$ overall) show a brown color.

Similarly, among those D-to-Z diamonds with a yellowish tint, that color is predominantly from cape-related defects. However, the defects are at such a low concentration that the diamond cannot attain a Fancy color grade.

Figure 3. The Vis-NIR absorption spectra for two Y-Z diamonds and two D-color diamonds. The type Ia diamonds show cape features that correspond with yellow coloration; the D-color type Ia sample also shows the contribution of N3 fluorescence, which reduces the appearance of the N2 absorption for that sample. The spectra of the two type II a diamonds show the brown absorption continuum, a relatively featureless absorption that increases toward lower wavelengths and becomes more prominent with stronger brown color.

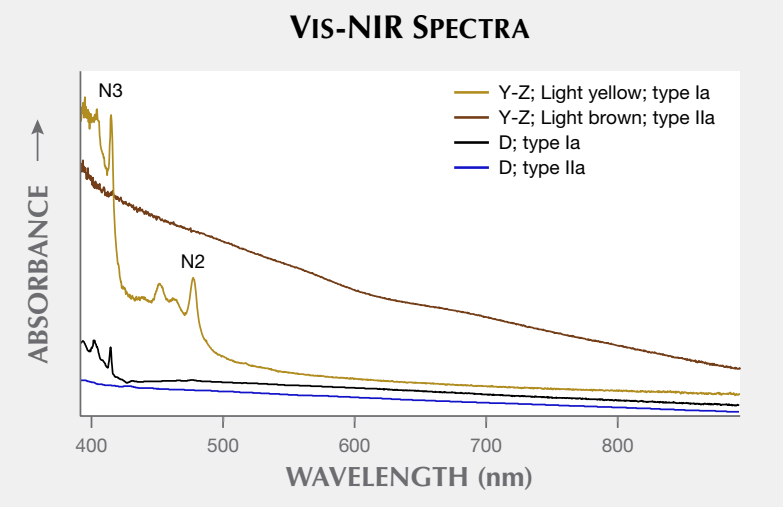

Brown. Though not as common as yellow in submissions to the laboratory, brown is more prevalent than most other diamond colors. The vast majority of $\mathrm{D}$ to-Z diamonds with brownish tints owe their color to selective light absorption due to vacancy clusters (i.e., clusters of vacant carbon-atom positions; see Hounsome et al., 2006; Fisher et al., 2009). These clusters, created through natural plastic deformation of the diamond lattice, are believed to contain approximately 40-60 vacancies that cause a gradually increasing absorption across the visible spectrum toward lower wavelengths (Fisher et al., 2009; Dobrinets et al., 2013; figure 3).

\section{OCCURRENCE AND FORMATION}

Yellow. Most diamonds grow within the earth's continental lithosphere (Shirey and Shigley, 2013). By the time they are faceted, there are typically no distinguishing features remaining from the geographic region in which they formed or from their transport to the earth's surface. The yellowish coloration in D-to$\mathrm{Z}$ diamonds generally arises from cape features such as the N3 center, which forms from a high amount of nitrogen incorporation (> $500 \mathrm{ppm}$ ) combined with the dual variables of geologic time and high temperature. The presence of nitrogen in the earth's lithosphere where diamonds grow, residence time scales of millions to billions of years, and temperatures of about $1100-1200^{\circ} \mathrm{C}$ (Shirey and Shigley, 2013) all lead to the 
transformation of single nitrogen to more aggregated nitrogen defects, and the accompanying formation of cape spectral features, in the vast majority of natural diamonds. Most of these show intermediate concentrations of color-causing nitrogen incorporation such as N3 and its related absorptions such as N2-neither so small that the diamond is colorless, nor so great that it achieves a Fancy yellow color grade.

Brown. Both brown and pink color in diamonds (due to the formation of the brown absorption continuum and the $550 \mathrm{~nm}$ absorption band) are believed to be created by plastic deformation. However, careful high-pressure, high-temperature (HPHT) annealing of brown-pink diamonds can reduce the brown color while preserving the pink color, indicating that the corresponding $550 \mathrm{~nm}$ absorption band has higher thermal stability. Therefore, it appears likely that the deformation producing pink color occurs inside the earth's mantle (Smit and Shor, 2017; Eaton-Magaña et al., 2018b), while the more prevalent brown coloration is likely caused by the diamond's rapid ascent to the surface in kimberlite or lamproite vulcanism (Gaillou et al., 2010).

Distinction by Diamond Type. Diamond type has proven to be an important distinguishing characteristic in fancy-color diamonds, and colorless diamonds are no exception (Breeding and Shigley, 2009). Box A illustrates the nitrogen configurations that lead to the different diamond types, as well as the importance of this classification for color origin determination in colorless to near-colorless diamonds.

Recent research on the inclusions contained within type IIa (Smith et al., 2016), type IIb (Smith et al., 2018), and some type IaB diamonds (Kagi et al., 2016; Rudloff-Grund et al., 2016; Gu and Wang, 2017) has demonstrated that many of these stones have a

Figure 4. Recent research has shown that some type IaB, many type IIa, and potentially all type IIb diamonds have superdeep origins below the continental lithosphere, while the majority of the world's diamonds (generally type Ia/IaAB, but also some type IIa) have a more shallow lithospheric origin ( 150-200 km depth; Shirey and Shigley, 2013). Most kimberlite pipes are found in geologically ancient and stable portions of the crust that geologists call cratons.

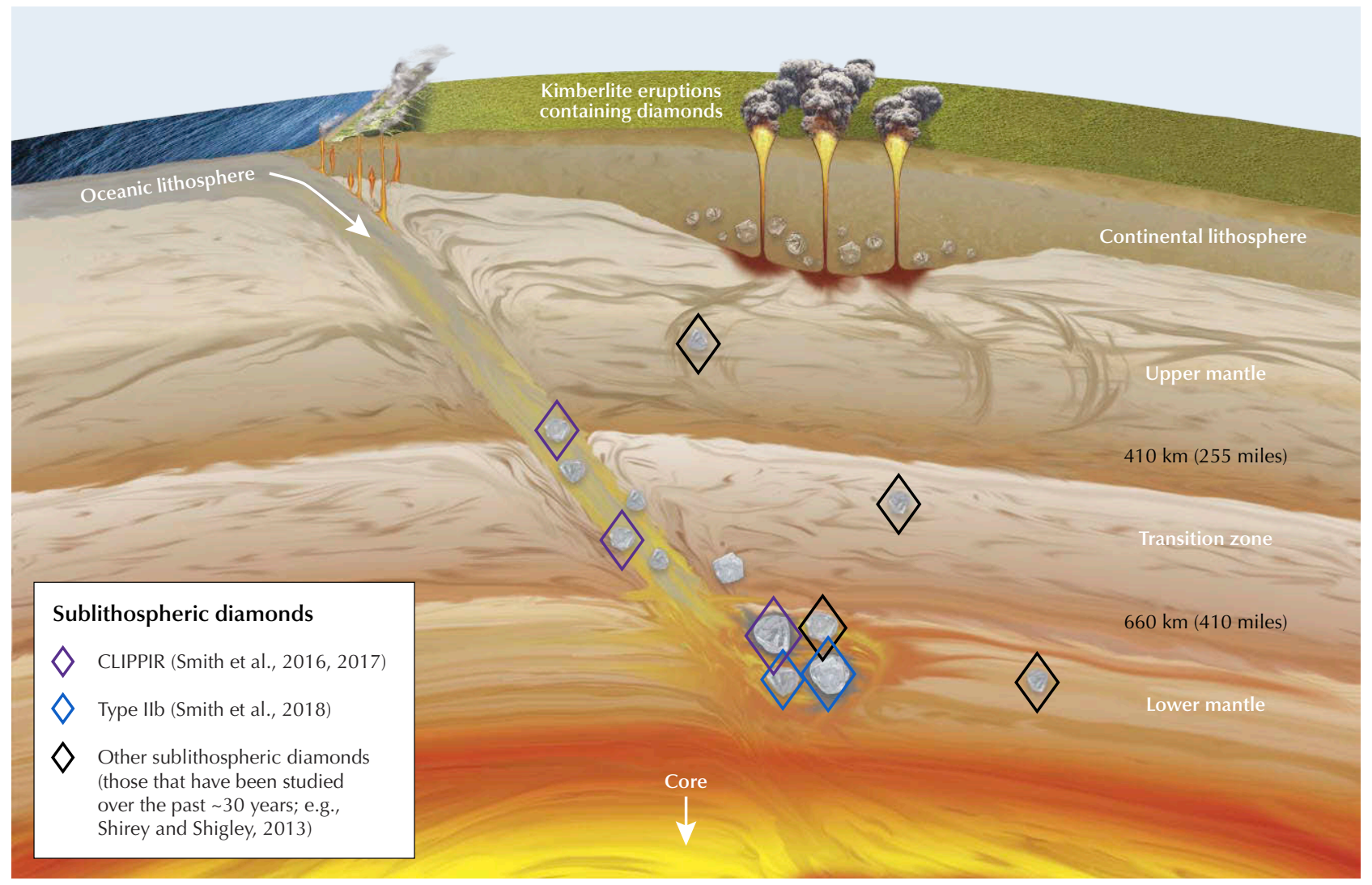




\section{Box A: A Brief Summary of Diamond TyPe}

One of the foundations of diamond science is the determination of diamond type. The presence/absence of nitrogen or boron distinguishes the range of color, defects, and potential treatment history. Recent research has also shown that the various diamond types can have very different geological formation methods and depths (Shirey and Shigley, 2013; Smith et al., 2016, 2017, 2018; Kagi et al., 2016; Rudloff-Grund et al., 2016; Gu et and Wang, 2017).

The B-aggregate has been identified as four nitrogen atoms surrounding a vacancy (Boyd et al., 1995), and it is generally considered the end product of the nitrogen aggregation process. Geologically young diamonds show nitrogen as single isolated atoms that coalesce over geologic time into pairs (A-aggregates; Kiflawi et al., 1994) and then ultimately into B-aggregates (figure A-1). This pairing of nitrogen atoms (and the subsequent steps of aggregation) involves internal diffusion at relatively high temperature and/or long geologic residence times during which the nitrogen atoms can move through the lattice by filling vacancies or changing places with adjacent car- bon atoms. During the next stage of aggregation, the A centers form B-aggregates, and there is the side reaction (Woods, 1986) that produces N3 centers (three nitrogen atoms with a vacancy). The A- and B-aggregates are infrared active and produce infrared spectral features but no features in the visible spectrum (i.e., they do not produce any color). Meanwhile, the N3 defect is the opposite-it has no infrared features but possesses visible spectral features that result in yellow coloration.

If all A-aggregates have converted to B-aggregates and formed a type IaB diamond, then generally the diamond is geologically quite old or was subjected either to higher temperatures that encouraged aggregation or a combination of long residence time and high temperature. However, the vast majority of type I diamonds show a combination of A- and B-aggregates, so very few D-to-Z natural diamonds $(0.09 \%)$ are shown to reach the type IaB endpoint.

Figure A-1 (bottom) shows another example of the importance of diamond types. The natural abundance of D-

Figure A-1. Top: The progression of nitrogen aggregation in natural diamonds. All young diamonds that contain nitrogen have it in the isolated form. Over millions to billions of years of residence in the earth, these isolated atoms find each other and pair to form A-aggregates. These A-aggregates further coalesce to form B-aggregates resulting in a combination of $A$ - and B-aggregates - most natural diamonds are at this point on the $A \rightarrow B$ pathway. Very few progress to complete aggregation and show only B-aggregates. Bottom: The diamond type distribution for several forms of $D$-to- $Z$ diamond demonstrates the importance of identifying whether a diamond is type II or type IaB, as these could be laboratory-grown or HPHT-treated.

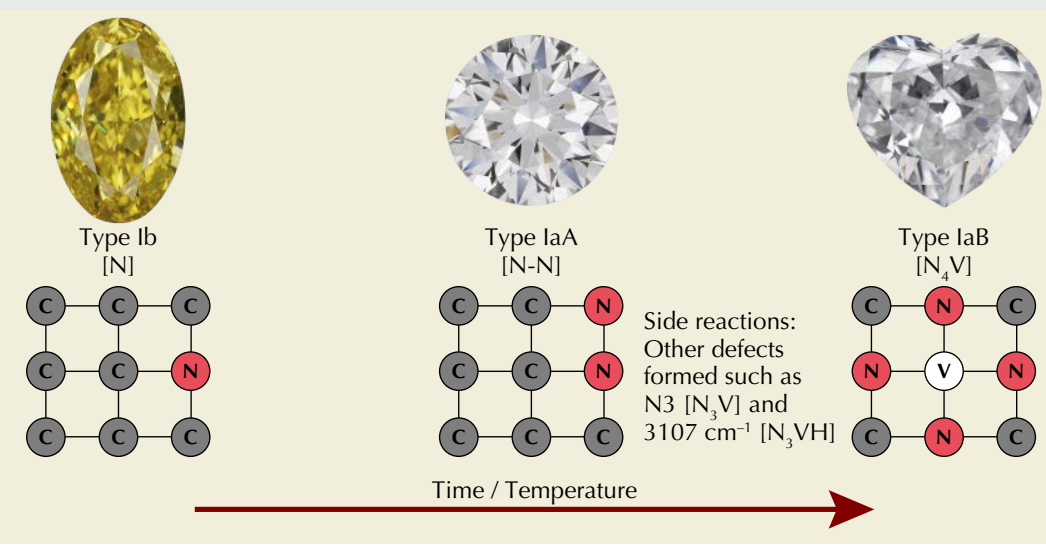

\section{D-to-Z Diamond Type Distribution}
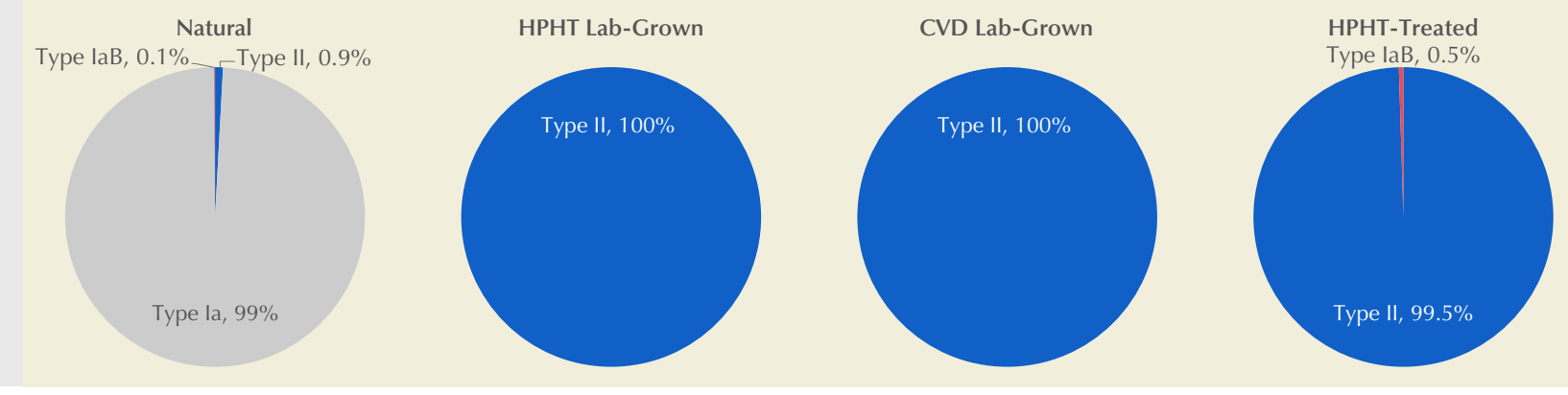


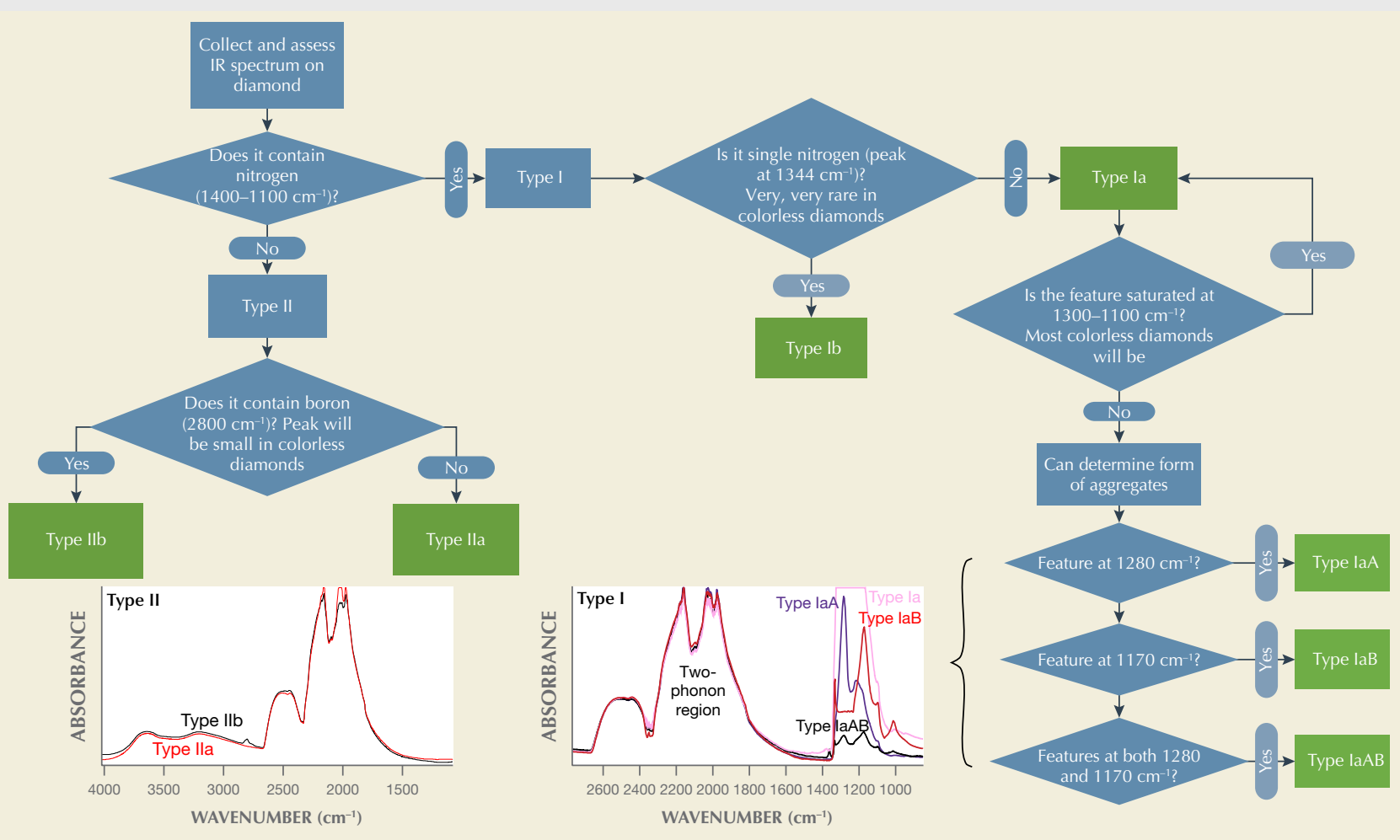

Figure A-2. This flowchart illustrates the identification process for determining diamond type.

to- $\mathrm{Z}$ diamonds is heavily skewed toward type $\mathrm{Ia} / \mathrm{IaAB}$ $(99 \%)$. Meanwhile, laboratory-grown diamonds in the Dto-Z color range are type II, while HPHT-treated D-to-Z diamonds can be either type II or type $\mathrm{IaB}$ (see box $\mathrm{B}$ for more regarding HPHT-treated diamonds). However, some reports on treated HPHT-grown diamonds indicate this could change in the future (Fang et al., 2018).

One aspect of comparing the infrared spectra of faceted stones is that the path length through a rough or polished diamond is unknown and rather difficult (or impossible) to determine compared to a double-sided parallel-polished plate, where the path length is simply the plate thickness. Fortunately, we can compare infrared spectra from different diamonds because the absorption coefficient of diamond is generally considered to be constant in the twoand three-phonon regions. For example, at $2000 \mathrm{~cm}^{-1}$ the absorption coefficient is $12.3 \mathrm{~cm}^{-1}$ (Tang et al., 2005; Breeding and Shigley, 2009). Each diamond IR spectrum, regardless of the sample's thickness or path length, can be compared by applying this normalization. As the twophonon region is often saturated in the IR spectra of faceted diamonds, we generally compare each IR spectrum using the three-phonon region and apply that normalization to determine nitrogen/boron concentrations.

An interesting side note regarding the naming of the $\mathrm{N} 3$ and $\mathrm{H} 3$ centers (among others) is that the "N" in N3 and the " $\mathrm{H}$ " in $\mathrm{H} 3$ do not refer to nitrogen or hydrogen. Instead, they refer to "natural" and "heated," (Zaitsev, 2003), as these defect centers were observed and named decades ago in the Vis-NIR absorption spectra of natural and heated diamonds. Researchers at the time designated the peaks across the spectrum as 1, 2, 3, etc. In the years since, diamond scientists have identified these configurations. We now understand their formation process and can also detect H3 in the Vis-NIR absorption and photoluminescence spectra of natural, unheated diamonds. Nevertheless, the defect nomenclature remains. The configuration of the $\mathrm{N} 3$ defect is $\mathrm{N}_{3} \mathrm{~V}$-it is pure coincidence that this defect contains three nitrogen atoms-and the $\mathrm{H} 3$ defect is NVN.

Similarly, type I and type II diamonds were distinguished decades ago, mainly on the basis of UV transparency (Robertson et al., 1933) instead of the detailed analysis of IR spectra used today. In the decades since that initial identification, scientists have recognized that the absence of nitrogen leads to observed UV transparency in type II diamonds, while the presence of nitrogen creates the UV absorption (type I). IR spectroscopy has allowed the distinguishing of the various forms of nitrogen and whether boron is present as the major impurity, to further subcategorize the two diamond types (figure A-2). 
"superdeep" origin. These form principally at depths of $\sim 300-800 \mathrm{~km}$, largely within the mantle transition zone and uppermost lower mantle, while the vast majority of diamonds form within the continental lithosphere at depths of 150-200 km (figure 4). The differences in pressure, temperature, and local chemistry at these very distinct formation depths produce many of the differences between these diamond types.

At the greater depths where type II diamonds form, nitrogen is presumably not available in the surrounding geologic environment. Therefore, it cannot be incorporated into the growing diamond as with type I stones. Superdeep type IaB diamonds are exposed to much higher temperatures and pressures than their lithospheric cousins, and those higher temperatures promote complete nitrogen aggregation (again, see box A or Breeding and Shigley, 2009, for more information). While many, if not most, type IIa diamonds may be assumed to have a superdeep origin, there are some known type IIa diamonds that originate from the lithosphere along with type Ia diamonds (Smith et al., 2017). It is important to note that the type classification scheme does not translate directly to a geological classification, even though some notable and meaningful correlations exist between diamond type and the various geological settings of formation.

\section{COMPARISON OF SPECTROSCOPY WITH LABORATORY GRADING}

Standard testing conditions and instrumentation are applied to all diamonds submitted to GIA. Some that are potentially treated or laboratory-grown undergo additional testing. The instrumentation has been detailed in other publications (e.g., Smit et al., 2018) and is summarized for this series of articles in Breeding et al. (2018). In this study, diamond type was automatically determined from the IR spectra using a mathematical algorithm similar to the one used by GIA's DiamondCheck instrument, and the result was verified by the technician. Among those determined as type Ia, the calculation of the collected spectra, particularly the nitrogen concentration (in the form of A- and B-centers) and the intensity of the $\mathrm{N}_{3} \mathrm{VH}$ defect $\left(3107 \mathrm{~cm}^{-1}\right.$ peak), was performed using custom software written by one of the authors (TA). The evaluation of these results was verified by manually calculating the aggregate concentrations on several hundred diamonds using a customized algorithm derived from a spreadsheet provided by Dr.
David Fisher (De Beers Group Technology, Maidenhead, UK) and comparing those results with the software's calculations. After the spectral results of all type Ia diamonds were calculated, the calculations were verified again on several hundred randomly selected diamonds. For the plots in this article, the dataset consisted of D-to-Z diamonds submitted to all GIA labs within a significant portion of 2017, as described above. A different dataset was used for the charts presented in box B, and those specifics are described therein.

Diamond Type and Grading Quality Factors. As noted above, diamond type is an important metric for inferring the likely formation depth of a diamond. It also creates a number of interesting comparisons when evaluating differences in grading quality factors. Box A provides a brief summary of diamond type; Breeding and Shigley (2009) examines this topic in much greater depth.

Because type II and type IaB diamonds comprise such a small percentage of submitted faceted diamonds, the dataset needed to be quite large so that these rare diamond types would have adequate representation, and therefore the dataset is composed of a majority of GIA's 2017 D-to-Z intake. Nevertheless, many of the following figures compare the distribution of grading quality factors for the various diamond types, even though the population within these groups may differ by several orders of magnitude. We show the distribution of the various grading quality factors for type II diamonds (separated into type IIa and boron-containing type IIb) and type I diamonds (separated as type IaB and type Ia/IaAB). To clarify, we use the designation of "type Ia" for all diamonds with saturated nitrogen (i.e., spectral absorption is too great to be quantified by the spectrometer, where we cannot distinguish the nitrogen aggregates more specifically) along with those that have unsaturated nitrogen and are determined to be either type IaA or type IaAB. These are also illustrated in box A. Among the many D-to-Z diamonds used for this study, $99.06 \%$ were determined to be type Ia/IaAB, $0.83 \%$ type IIa, $0.09 \%$ type $\mathrm{IaB}$, and $0.02 \%$ type $\mathrm{IIb}$. Figure 5 plots the distribution of various grading quality factors and fluorescence observations for type $\mathrm{Ia} / \mathrm{IaAB}$ stones, and figure 6 plots the distribution for the remaining diamond types. Type Ib diamonds are not included in these figures, as the isolated nitrogen defect $(\mathrm{N})$ associated with this diamond type generally introduces sufficient color to be classified as fancy color. 


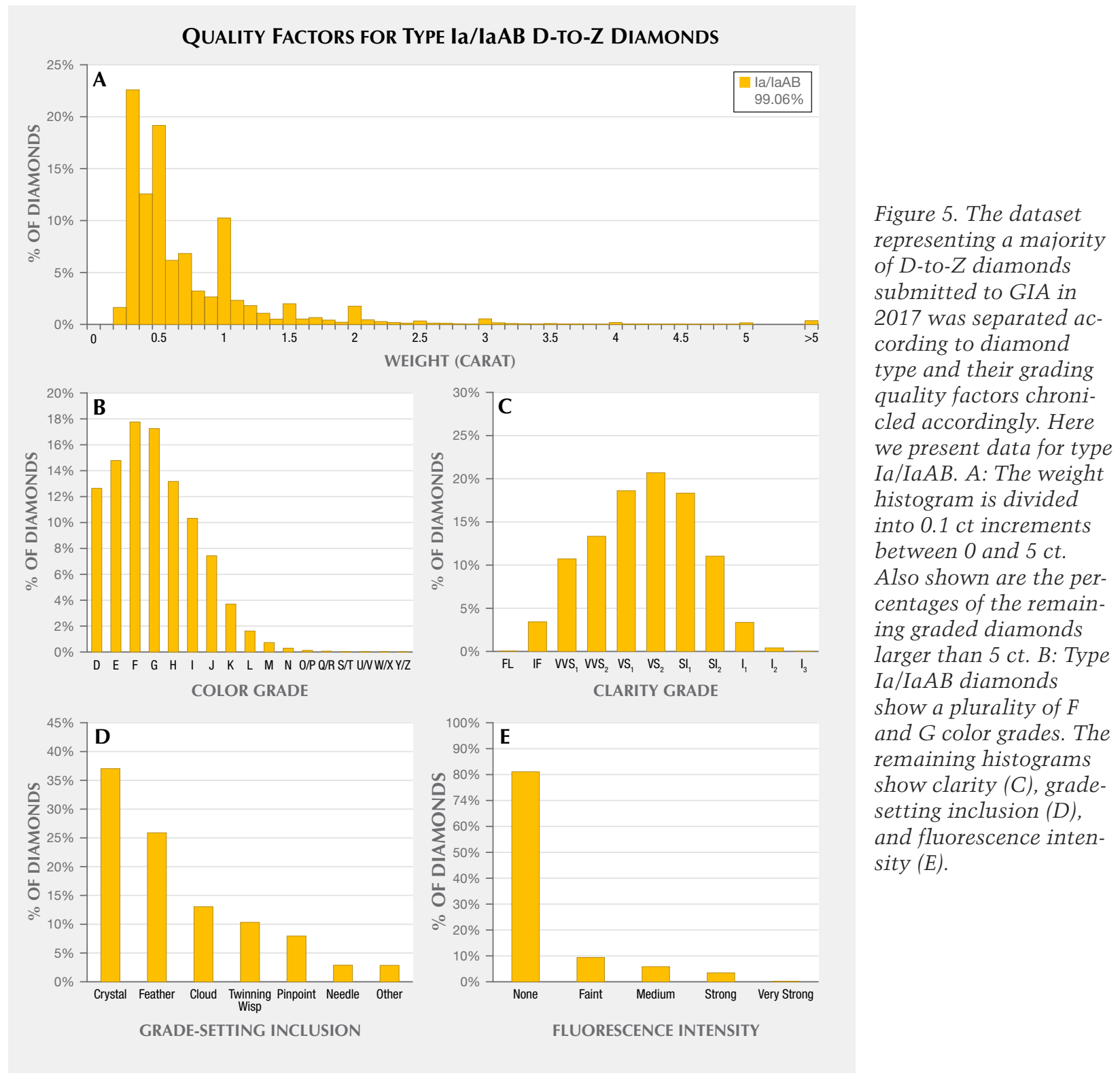

Weight. Figures 5A and 6A show the distribution of D-to-Z diamonds across the carat weights for type I and type II. The data are presented in 0.1 ct increments, and noticeable spikes in quantity are observed (not surprisingly) near important carat-weight thresholds (e.g., $0.25,0.5,1.0,1.5$, and $2.0 \mathrm{ct}$ ), particularly for type I diamonds. These figures also show that type Ia diamonds are more plentiful among the smaller carat weights, while type II are more plentiful at higher weights - a trend consistent with prior observations (Smith et al., 2017). For example, 34\% of type IIa diamonds weighed $0.5 \mathrm{ct}$ or less and $60 \%$ weighed $1 \mathrm{ct}$ or less. Among type $\mathrm{Ia} / \mathrm{IaAB}$ diamonds, $49 \%$ weighed 0.5 ct or less and $79 \%$ weighed 1 ct or less.
Color. Figures $5 \mathrm{~B}$ and $6 \mathrm{~B}$ plot the distribution of D-to$\mathrm{Z}$ color grades for the various diamond types. For type II and type IaB diamonds, the most common color is $\mathrm{D}$, and the percentages decrease as the color grade increases. For type $\mathrm{IIb}, 72 \%$ of those within the D-to- $\mathrm{Z}$ range are $\mathrm{D}$-color. These stones have a low boron concentration $(<100$ parts per billion); high boron values would generally place a diamond in the gray-to-blue fancy color range (King et al., 2008; Eaton-Magaña et al., 2017). Since type Ila diamonds have no detectable single nitrogen or nitrogen aggregates in the infrared, which can also correlate with nitrogen-related defects in the visible region, their major cause of color is plastic deformation, which gives a brown coloration. 
QUALITY FACTORS FOR TYPE Ila/IIb/laB D-TO-Z DIAMONDS
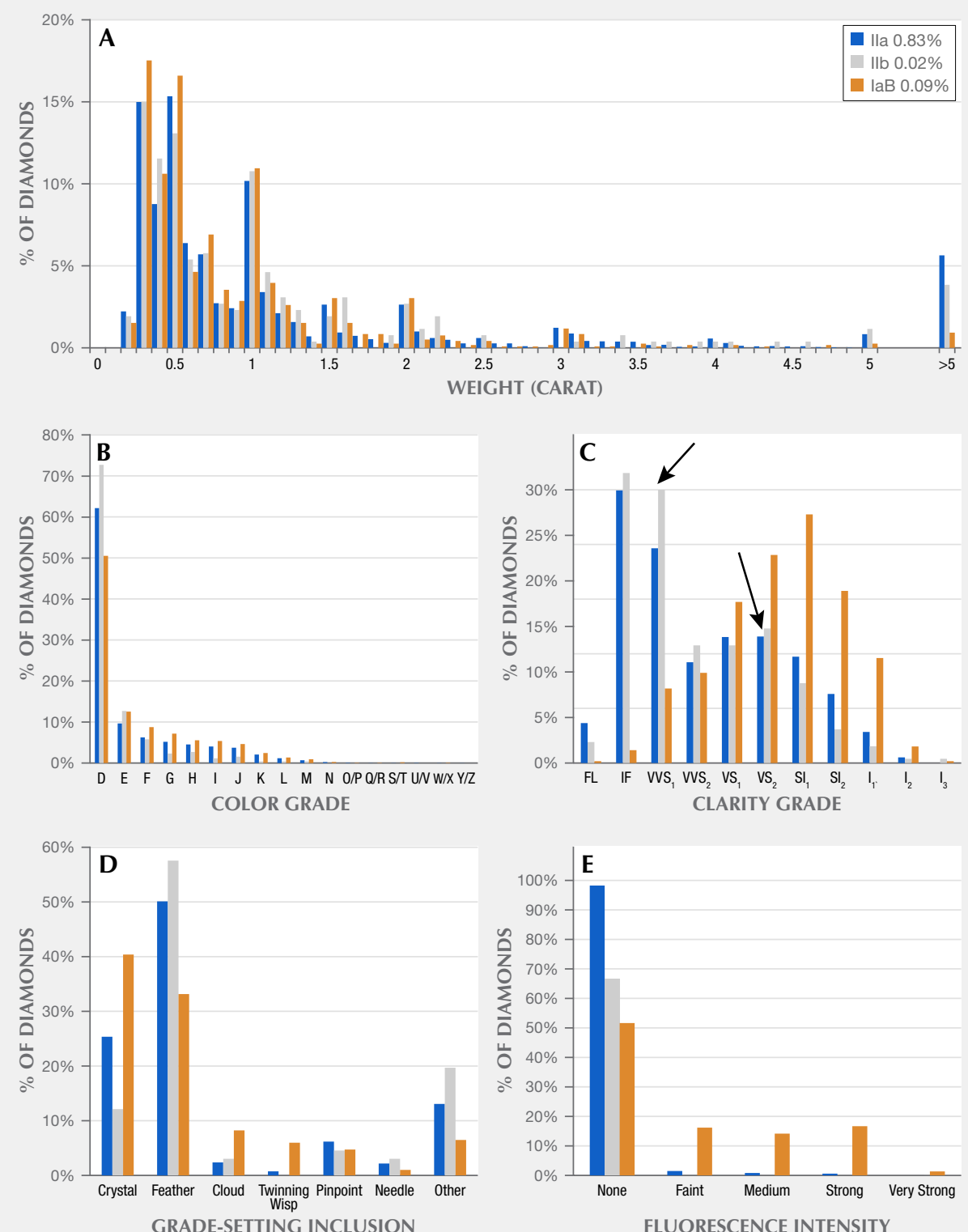

GRADE-SETTING INCLUSION

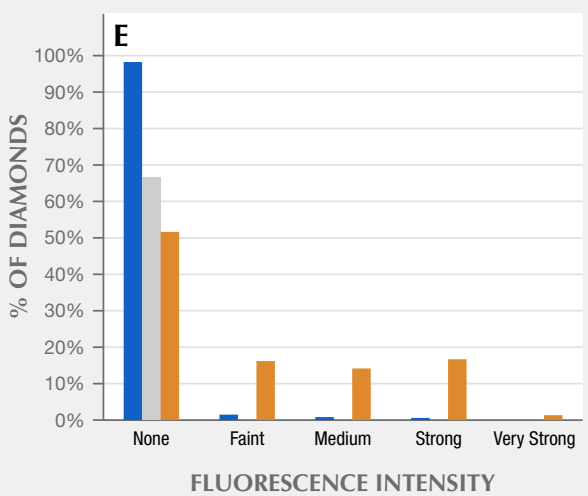

Figure 6. The dataset of Dto-Z diamonds was separated according to diamond type and their grading quality factors chronicled accordingly. Here we present data for type IIa/IIb/IaB. A: The weight histogram is divided into $0.1 \mathrm{ct}$ increments between 0 and $5 \mathrm{ct}$. Also shown are the percentages of the remaining graded diamonds larger than $5 \mathrm{ct}$. B: The color distribution for type II and type IaB diamonds shows that the majority are $D$ color. C: For type IIa and type IIb diamonds, a bimodal distribution is seen with maxima at IF/VVS clarity ranges and the $V S_{2}$ clarity grade (also marked by black arrows). This pattern noticeably contrasts with type I diamonds, which show a single maximum at the $V S_{2} / S I_{1}$ clarity range. $D$ : For the majority of type II diamonds, the grade-setting inclusion feature is a feather, with a relatively low incidence of both crystals and twinning wisps compared to type I. E: Type II diamonds have the highest number with no fluorescence response, while type IaB show the lowest percentage.
Among type Ia diamonds, the color grades of $\mathrm{F}$ and $\mathrm{G}$ show the highest population (figure $5 \mathrm{~B}$ ). Since type Ia is by far the most abundant diamond type, this color distribution represents the vast majority of D-to-Z diamonds submitted to GIA for grading. Again, for type Ia diamonds, the major causes of color are higher concentrations of nitrogen-related defects that increase the yellow coloration.

Figure 7A shows the distribution of $G$ to $Z$ grades and the percentages for each color grade for brown versus yellow coloration; the brown/yellow assessment was made using GIA's internal color grading methodology. For G-color diamonds, only
$5 \%$ had brown coloration. For the combined-grade diamonds $(\mathrm{O}$ to $\mathrm{Z}$ that are presented as O-P, Q-R, S$\mathrm{T}$, etc.), the percentage showing brown coloration has increased to $16 \%$. It is interesting to note that for diamonds beyond the D-to-Z scale-that is, unmodified brown or yellow diamonds on the fancy color scale-there is a decidedly reverse trend, as there are far fewer Fancy brown than Fancy yellow diamonds submitted for grading, most likely due to market forces.

This trend in D-to-Z diamonds toward an increasing proportion of brown coloration with higher color saturation is also seen in figure $7 \mathrm{~B}$, which plots the 
average nitrogen concentration of B-aggregates with color grade in type IaB diamonds. From D to G color, we see an increase in the concentration of B-aggregates, reaching a maximum for $\mathrm{G}$ color. For the combined-grade range ( $\mathrm{O}$ to $\mathrm{Z})$, the average nitrogen concentration (across all diamonds in the dataset) decreases as the percentage of diamonds with brown coloration becomes more prominent.

Figure $7 \mathrm{C}$ shows the results of the automatic calculations for nitrogen-aggregate defect concentrations from the infrared spectra for type Ia/IaAB diamonds.
Diamonds on the D-to-Z scale are predominantly type Ia with a mixture of A- and B-aggregates; many of them have such high nitrogen concentrations that we cannot determine the $\mathrm{A} / \mathrm{B}$ ratio or the exact concentration (because the intensity of the infrared peaks exceeds the vertical scale of the spectrum plot due to very strong absorbance in this region). We generally refer to these as saturated type Ia or often as simply "type Ia." For faceted diamonds analyzed with current infrared spectrometers, those with nitrogen concentrations greater than 500 parts per million generally have infrared spec-

Figure 7. A: The yellow or brown coloration is shown for $G$ to $Z$ color. The percentage of diamonds with brown coloration increases from $5 \%$ for $G$ color to $16 \%$ for the combined grades (O to $Z$ ). B: Among the small quantity of type I diamonds determined as type IaB, the concentration of B-aggregates can be determined and plotted versus color. The quantity increases from $D$ to $G$ color and then decreases as the brown coloration in these type IaB diamonds becomes more pronounced. C: The vast majority of type Ia/IaAB diamonds have IR spectra with a saturated nitrogen region, making it impossible to calculate their nitrogen concentration or $A / B$ aggregate ratio. D: The $3107 \mathrm{~cm}^{-1}$ peak $\left(N_{3} \mathrm{VH}\right)$ in the IR spectra can be determined in diamond, even if the nitrogen region is saturated. Here, the calculated median value and the values for the upper and lower quartiles illustrate the range of values for each color grade.

A

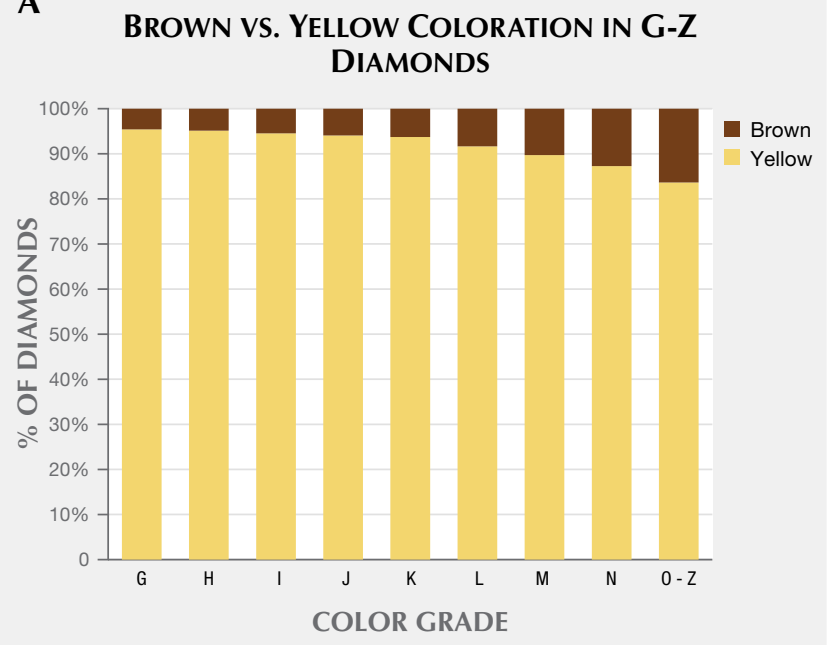

C

SATURATED Nitrogen AGgReGATES IN IR SPECTRA

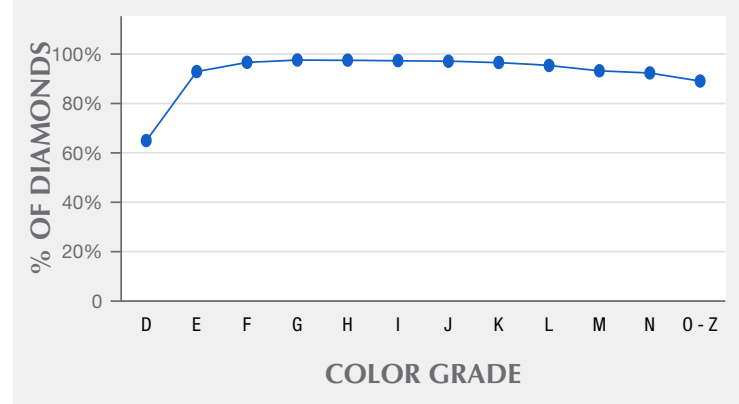

B

\section{AVerage Nitrogen B-Aggregate Concentration IN TYPE IaB DIAMONDS VS. COLOR GRADE}

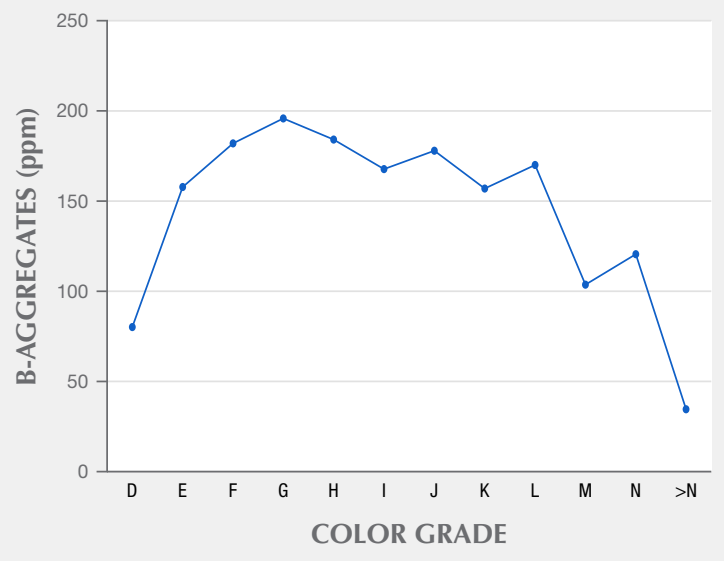

D

NORMALIZED $3107 \mathrm{~cm}^{-1}$ PeAK AREA IN IR SPECTRA FOR TYPE Ia D-TO-Z DIAMONDS

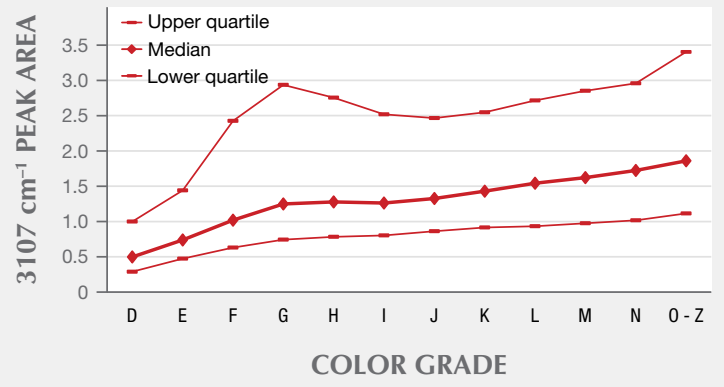




\section{Box B: Grading Quality Factors in HPHT-Treated Diamonds}

We discuss here a dataset that is different from the one in the main body of the article. Here, the dataset consists of HPHT-treated diamonds in the D-to- $Z$ range that have been submitted to all GIA laboratories over the last decade; it includes several thousand stones.

Gemological data have been published on small quantities of stones when this treatment was first seen in the trade (Chalain et al., 1999; Fisher and Spits, 2000) and when notably large diamonds were HPHT treated (e.g., Wang, 2010). As mentioned in the Identification section, only type II and the rare type IaB diamonds may be HPHT treated to remove or reduce brownish or grayish coloration. Most commercial HPHT treatments are conducted in the $1900-2100^{\circ} \mathrm{C}$ range, as reaching these temperatures is considered relatively inexpensive and reliable for the color treatment of light brown diamonds (Dobrinets et al., 2013).

The spectra and images for our dataset of HPHTtreated diamonds were collected using similar methods to those described above for natural-color D-to-Z diamonds. The same software was used to assist in diamond type determination and nitrogen concentrations for type $\mathrm{IaB}$ diamonds. Among this dataset of HPHT-treated diamonds, $0.5 \%$ were type $\mathrm{IaB}, 1.7 \%$ were type $\mathrm{IIb}$, and the balance $(97.8 \%$ ) were type IIa. Among the type IaB diamonds chosen for HPHT treatment, the concentration of $\mathrm{B}$-aggregates is considerably lower than among natural, untreated type IaB. The HPHT-treated, type IaB diamonds had B-aggregates ranging from $\sim 1$ ppm to $90 \mathrm{ppm}$, with an average value of $<10 \mathrm{ppm}$. In comparison, the Baggregates in natural, untreated type IaB ranged from $\sim 1$ ppm to $570 \mathrm{ppm}$, with an average value of $\sim 120 \mathrm{ppm}$.

Figure B-1 illustrates the distribution of various grading quality factors for these stones. Unlike the data in the main body of this article, these graphs plot the different diamond types together due to the much smaller sample set. A comparatively large percentage of these stones $(11 \%)$ are greater than $5 \mathrm{ct}$, almost twice the percentage we see among natural type IIa diamonds $16 \%$; compare figure B-1A with figure $6 \mathrm{~A})$. It is possible that the larger-size, off-color diamonds were considered worth the risk and expense of the treatment to improve their color.

Figure B-1B shows the post-treatment color distribution of these HPHT-treated diamonds. The majority are heated to high temperatures $\left(1900-2100^{\circ} \mathrm{C}\right.$; Dobrinets et al., 2013) such that the result is in the colorless range (D to $\mathrm{F})$. Figure $\mathrm{B}-1 \mathrm{C}$ shows the shape distribution of HPHT-treated diamonds. Figure B-1D shows the clarity distribution of HPHT-treated diamonds, which, as with natural type II diamonds, skews toward the higher clarity grades but does not show the bimodal distribution seen among the natural type IIa diamonds (figure 6C). Despite the high stabilizing pressures used in HPHT treatment, diamonds with poorer clarity grades and a number of internal clarity features generally do not benefit from HPHT treatment due to discoid fractures that form around crystal inclusions and graphitization of preexisting fractures (Fisher and Spits, 2000). For a majority of these HPHT-treated diamonds, a feather is the grade-setting feature (figure B$1 E$ ), which is comparable with the natural distribution of type IIa diamonds (figure 6D). Yet the incidence of crystal inclusions is far lower in HPHT-treated diamonds $(10 \%$ compared to $25 \%$ for IIa natural diamonds), showing that type IIa diamonds are carefully chosen for HPHT processing to minimize the possibility of adverse effects from this treatment process. tra with saturated nitrogen. The likelihood that an IR spectrum will be saturated is also affected by the size of the faceted diamond as the path length approximately increases with size for the same shape. A thin plate fashioned from a type Ia diamond would have a higher threshold for saturation, and these higher nitrogen concentrations could be quantified.

Figure $7 \mathrm{C}$ shows that the vast majority, nearly $100 \%$ for most color grades, have saturated spectra in which the A- and B-aggregate concentrations cannot be determined. The exceptions are D-color diamonds, in which only $65 \%$ have saturated nitrogen. There is a slight decrease in the percentage of lower color grades (moving toward Y-Z color) showing saturated spectra; the higher number of diamonds showing brown coloration decreases the overall average nitrogen concentration.
Since the vast majority of type Ia/IaAB D-to-Z diamonds have saturated nitrogen in their infrared spectra, we are unable to determine their nitrogen concentration or their $\mathrm{A} / \mathrm{B}$ aggregate ratio. Therefore, any trends in total nitrogen or $\mathrm{A} / \mathrm{B}$ ratio can include only a small percentage of diamonds where the infrared spectral features fall within the vertical scale of the spectrum plot. In order to observe trends in the infrared spectra of this massive database without being able to look at nitrogen aggregates, we can consider other features in the infrared-most notably the 3107 wavenumber $\left(\mathrm{cm}^{-1}\right)$ peak. This peak has long been correlated with hydrogen impurity in diamond, and it has been recently attributed to the $\mathrm{N}_{3} \mathrm{VH}$ defect-that is, the N3 center plus a hydrogen atom (Goss et al., 2014). N3 is an important color center in $\mathrm{D}$-to-Z diamonds. It is one of the major defects 


\section{QUALITY FACTORS FOR HPHT-TREATED D-TO-Z DIAMONDS}
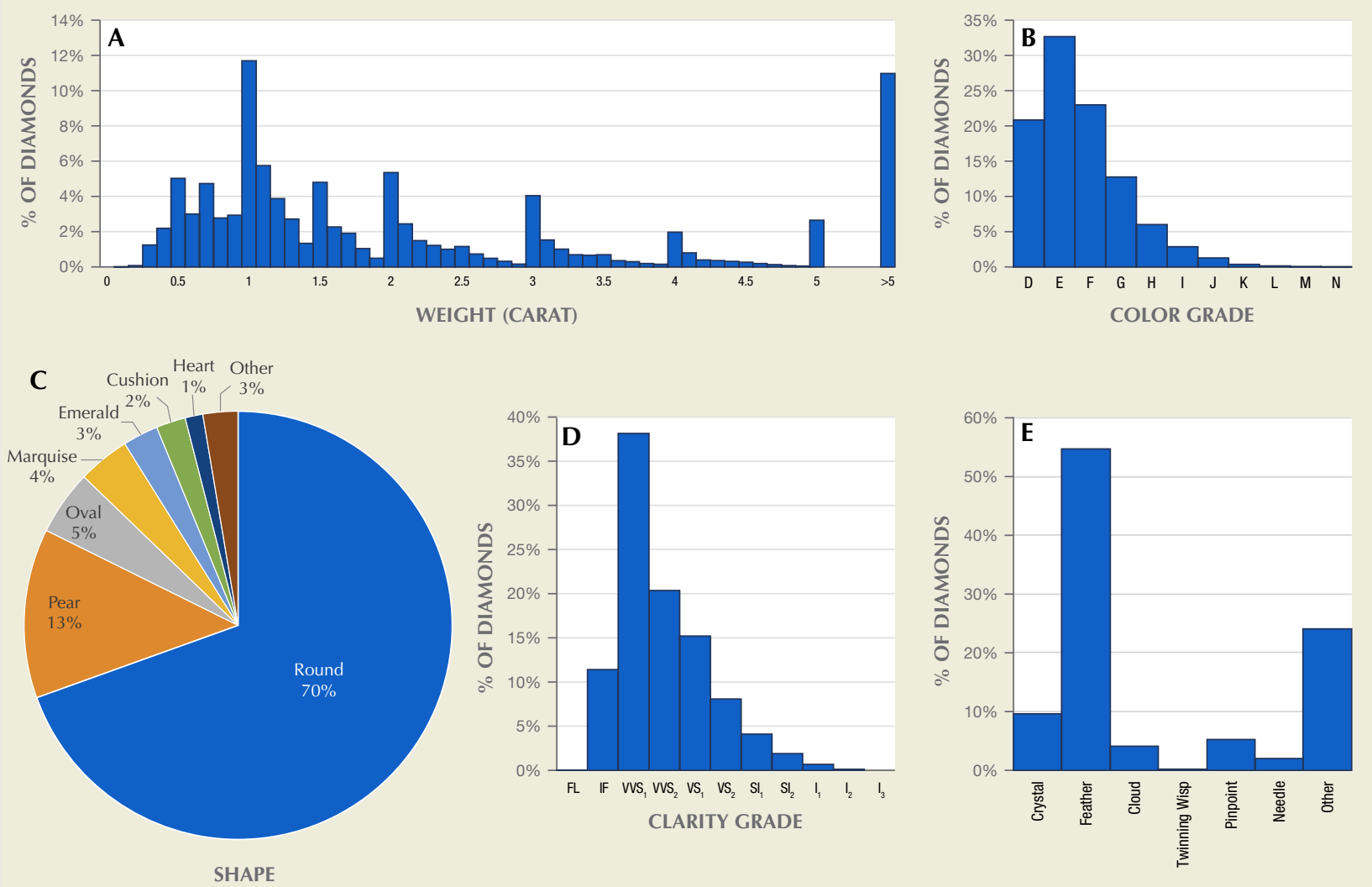

GRADE-SETTING INCLUSION

Figure B-1. The distribution of grading quality factors in D-to-Z HPHT-treated diamonds are shown for weight (A), color (B), shape (C), clarity (D), and grade-setting clarity characteristic (E).

leading to yellow coloration, and it creates the most commonly observed blue fluorescence. The $3107 \mathrm{~cm}^{-1}$ peak rarely saturates within the spectrum, so its intensity can be determined for almost all infrared spectra, including those with saturated nitrogen-aggregate spectral features, although its intensity may not always correlate with other nitrogen defects. Figure 7D presents the results of these calculations for the normalized $3107 \mathrm{~cm}^{-1}$ peak area (which is generally proportional to the concentration) plotted versus the graded color (see box A for more detail on this normalization). The values represent the median within each color grade, while the upper and lower quartile values indicate the spread of data for these diamonds. The graph shows that the median value of the $3107 \mathrm{~cm}^{-1}$ intensity increases with color. The upper quartile (i.e., those diamonds with values be- tween the median and the 75th percentile) also shows a high value at around G color, possibly due to the very high nitrogen seen in those diamonds.

Clarity. One interesting trend among the various diamond types (figures 5C and 6C) is that type II diamonds have a bimodal clarity grade distribution - that is, they show a maximum in percentage of diamonds with IF/VVS clarity grades and another maximum in $\mathrm{VS}_{2}$ clarity (indicated by arrows on the graph). This pattern is seen independently in both type Ila and type IIb. Meanwhile, type I diamonds (both type Ia/IaAB and type $\mathrm{IaB}$ ) show one maximum at $\mathrm{VS}_{2} / \mathrm{SI}_{1}$ clarity grades. While type IaB diamonds share a similar pattern in color grade distribution with type II diamonds (see again figure 6B), they show a similar clarity grade distribution with type Ia diamonds in figure 5C. 
Figures 5D and 6D show the distribution of gradesetting clarity characteristics for the different diamond types. Since many of the type II diamonds skew toward the higher clarity grades, the clarity features are likely smaller and less abundant/noticeable than in their type Ia/IaB counterparts. Nevertheless, the most common clarity characteristic in type II diamonds is feathers, with a much smaller percentage of crystal inclusions than in type I. This comparison is consistent with the prior observation of Smith et al. (2017) regarding superdeep type IIa diamonds, which they termed CLIPPIR diamonds-an acronym that contains the phrase "inclusion-poor." Also, the twinning wisp clarity feature is much less abundant among type II than type I diamonds $10 \%$ in type $\mathrm{Ia} / \mathrm{IaAB}$ diamonds versus $0.7 \%$ in type IIa and $0 \%$ in type $\mathrm{Ib})$. Twinning wisps are groups of microscopic inclusions formed along an orientation change in the crystal structure (a twin plane) during growth. These are considered unique to natural diamonds and, as seen here, mostly type Ia natural diamonds.

To study the bimodal distribution of clarity grades in type II diamonds in greater detail, we examined the grade-setting inclusions. The results show that among type Ila and type $\mathrm{IIb}$ diamonds with $\mathrm{VVS}_{1}$ clarity, feathers were the most common grade-setting inclusions, while other inclusions such as pinpoints or clouds were seldom the grade setters. At lower clarity grades in type IIa diamonds, feathers or crystals were the most common. For a grade-setting feather to be considered $\mathrm{VVS}_{1}$, it must be small, located on the pavilion, and most importantly have little to no depth; often these can be improved to IF by repolishing. Therefore, type II diamonds appear to have two distinct clarity populations-about half $48 \%$ of type $\mathrm{IIa}$ and $53 \%$ of type IIb) are FL or IF, or VVS, that are potentially improvable to IF. The other half generally have more significantly sized feathers, crystal inclusions, or other features, spanning from $\mathrm{VVS}_{2}$ to $\mathrm{I}_{1}$ clarity. Subsequent analysis of the clarity distribution in type II diamonds across the years 2010-2019 showed that this bimodal distribution was consistent and not specific to 2017.

If we limit our examination to only those stones larger than $10 \mathrm{ct}$, we see other interesting observations and distinctions between type I and type II diamonds. For large type II diamonds, the bimodal distribution does not remain. Instead, the majority of large type II diamonds are FL to $\mathrm{VVS}_{1}$, which is consistent with figure 2C in Smith et al. (2017). For such large diamonds, cutters are quite diligent in planning around inclusions within the rough. For ex- ample, the 603 ct Lesotho Promise rough showed several dark and likely metallic eye-visible inclusions and yet, through careful preparation and skilled cutting, yielded 26 D-FL diamonds $0.55-75$ ct totaling 224 ct; Krawitz, 2008). Meanwhile, type Ia/IaAB diamonds larger than $10 \mathrm{ct}$ show a clarity distribution similar to the entire dataset with a maximum at $\mathrm{VS}_{2}$ clarity grades, consistent with figure $2 \mathrm{C}$ in Smith et al. (2017).

While planning and cutting decisions help improve the clarity grade of faceted diamonds and guide the polisher to an IF/VVS grade, this does not generally appear to be possible for type Ia, even among higher-weight stones. It appears specific to a significant portion of type II rough.

Fluorescence. While fluorescence is not a grading quality factor, its observation is reported on grading reports and included here as well. Figures $5 \mathrm{E}$ and $6 \mathrm{E}$ show the distribution of fluorescence intensities among the various diamond types. As expected for type II diamonds, which have no detectable nitrogen in the infrared spectrum, they have very little incidence of fluorescence. In nearly $100 \%$ of these, fluorescence is described on the grading report as "None."

In contrast, type $\mathrm{IaB}$ diamonds have only $~ 50 \%$ with "None." This is not surprising, as the primary cause of fluorescence is the N3 defect, which is created as A-aggregates progress to B-aggregates (again, see box A). Additionally, type IaB diamonds do not contain A-aggregates that could act to quench (suppress) fluorescence, so type IaB diamonds show the highest incidence of medium to strong fluorescence. In diamonds containing A-aggregates, these interact with N3 defects in such a way that fluorescence may not be observed. The presence of A-aggregates inhibits fluorescence of the N3 center by creating an alternate, non-radiative (i.e., non-fluorescing) pathway for the electron to return from the excited state to the ground state (Collins, 1992; Vasil'ev et al., 2004).

Figure 8 plots the calculated features from the infrared spectra against the fluorescence intensity observations. As mentioned above, the vast majority of type Ia diamonds have saturated response in the nitrogen region of their infrared spectra, which makes it impossible to determine their concentration of nitrogen aggregates. In a small percentage of type Ia diamonds, we can determine the A- and B-aggregates and correlate those with the fluorescence intensity. Figure 8 (left) shows the median values for the A- and B-aggregates (and the upper and lower quartiles) for each fluorescence intensity. As the intensity pro- 


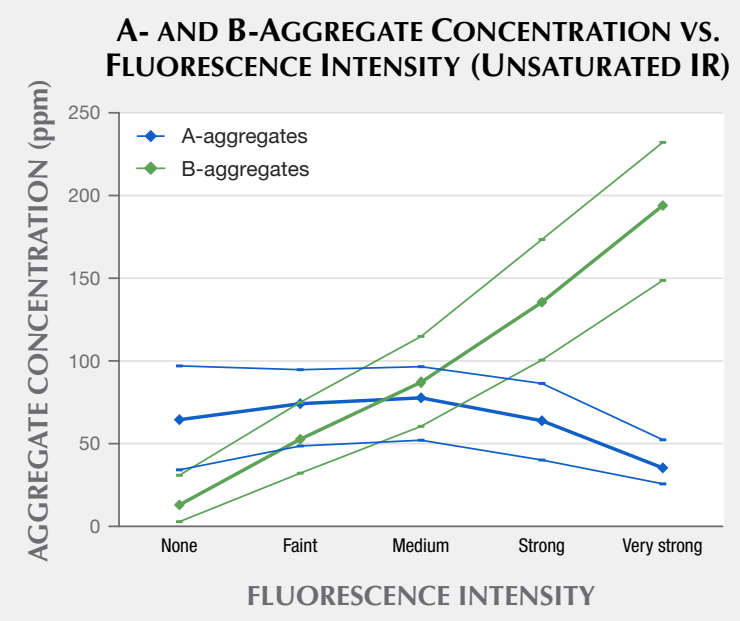

$3107 \mathrm{~cm}^{-1}$ NORMALIZED PEAK AREA vS. FLUORESCENCE INTENSITY

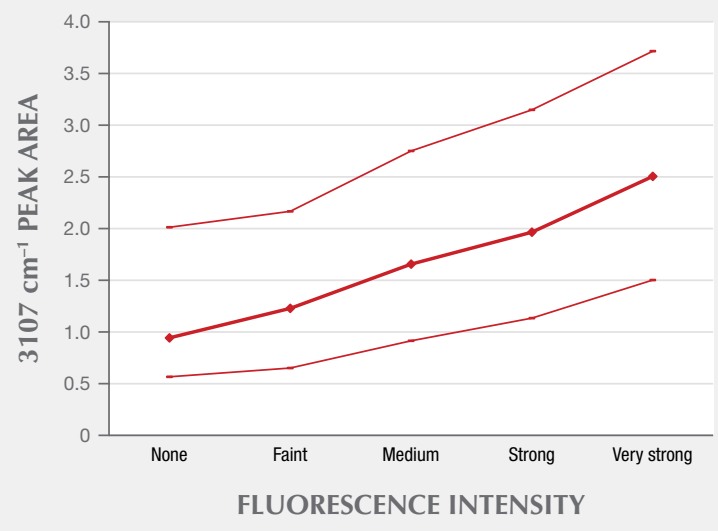

Figure 8. Left: For diamonds with unsaturated nitrogen, the A-and B-aggregates were calculated and plotted according to their fluorescence observations. For both $A$ - and B-aggregates, the median value along with the upper and lower quartile are plotted. Right: For a far higher number of the IR spectra for diamonds, the $3107 \mathrm{~cm}^{-1} \mathrm{normalized}^{-}$ peak area, the median value, and the upper and lower quartile can be plotted.

gresses, there is a shift from predominantly A-aggregates among the "None" category to predominantly B-aggregates among the "Very strong." Figure 8 (right) shows the increase in the $3107 \mathrm{~cm}^{-1}$ normalized peak area of the infrared spectra (again, the $3107 \mathrm{~cm}^{-1}$ peak is the N3 fluorescence defect associated with hydrogen; see Goss et al., 2014). The median value for the $3107 \mathrm{~cm}^{-1}$ normalized peak area increases from $\sim 1.0$ for those showing "None" to 2.5 for "Very strong."

\section{OTHER FACTORS: SHAPE AND CUT}

Software introduced shortly after 2000 has allowed manufacturers to plan the cutting of rough shapes with high color and clarity by mapping their inclusions. Cutters now use sophisticated planning pro- grams that maximize the cut quality (with specific parameters for various cut shapes) and clarity grades so that the highest-value yield guides the cutting choices. The software lists the options available in value order, and the cutter can then view instructions for where to saw the rough and parameters to cut in order to yield the highest value from the entire piece.

The morphology of the original rough also influences the shapes available to cutters. The octahedron, generally sourced from the continental lithosphere, is the most common shape for diamond rough. Figure 9 (left) shows that two round brilliants are often the best use of the available rough octahedron when planning. In contrast, irregular rough can often present a variety of shapes (such as rounds and a pear in the example in figure 9, right) as the best choices to the cutters.
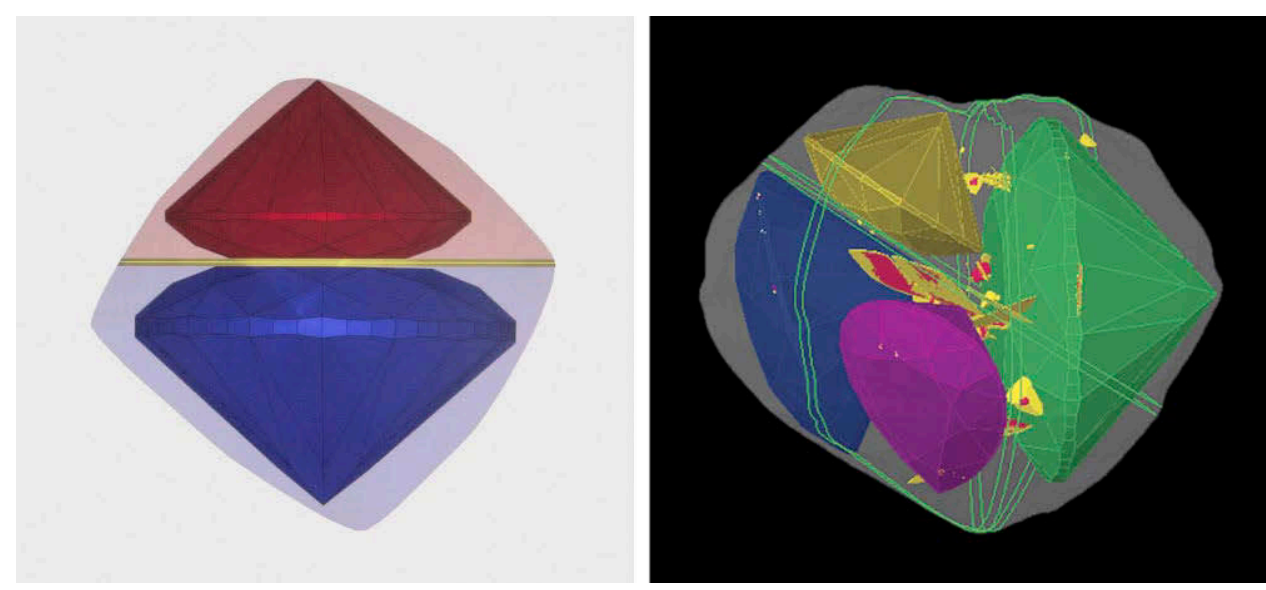

Figure 9. Left: The computer-generated planning solution for faceting a rough diamond octahedron into two rounds. This shape of rough is only expected to occur in diamonds originating from the lithosphere. Right: An irregular rough crystal (generated by Lexus M-Box software) can often lend itself to a variety of resulting shapes. 


\section{GEMOLOGICAL OBSERVATIONS}

Under magnification, the most prominent visible features in D-to-Z diamonds are mineral inclusions (crystals and clouds), feathers, twinning wisps, and graining. Numerous resources detail the various crystal inclusions and the microscopic world of natural diamonds (e.g., Koivula, 2000; Renfro et al., 2018). Among the most common mineral inclusions are garnets, olivines, clinopyroxenes, and various sulfide minerals, identified by their appearance and distinctive Raman spectra.

Graining, however, is not as widely understood as crystal inclusions. Graining in D-to-Z diamondsparticularly whitish, reflective, and transparent graining - was discussed by King et al. (2006) and includes any planar (or visibly linear and repeating) internal features seen under magnification (figure 10A). These features are widely considered to be planes of misaligned carbon atoms in the crystal structure, usually due to plastic deformation. In its more extreme forms, graining is associated with vacancy clusters that absorb light and produce a brown color (figure 10B). Brown graining is common in type Ia brownish diamonds in the $G$ to $Z$ range, but not as prevalent in type IIa diamonds with similar colors. When vacancy clusters are not sufficiently concentrated in the grain lines to produce brown color, the graining appears transparent or colorless and may be whitish or reflective, or even exhibit interference colors in rare instances due to distortion of the light by the planar features (figure 10, C and D). Even HPHTprocessed diamonds that have been decolorized often retain clearly visible transparent graining that was once brown before the vacancy clusters were destroyed by the treatment (figure 10E).

\section{IDENTIFICATION CONCERNS}

The vast majority of natural $\mathrm{D}$-to-Z diamonds are type Ia/IaAB (99\%; figure A-1) and cannot be decolorized by HPHT processing. Natural diamonds in the $\mathrm{D}$-to-Z range that are type IIa $(0.83 \%)$, type IIb $(0.02 \%)$, or type $\mathrm{IaB}(0.09 \%)$ can be subjected to HPHT processing to reduce a brownish or grayish appearance, and the color origin of such diamonds should be confirmed by a gemological laboratory (Chalain et al., 1999; Fisher and Spits, 2000). More information regarding HPHT-treated diamonds is discussed in box B.

For a colorless diamond to be grown by chemical vapor deposition (CVD) or HPHT methods, its diamond type must be type II (e.g., see figure A-1, top). An infrared spectrometer can quickly determine diamond type, helping to separate the vast population of colorless natural type Ia diamonds from the much smaller population of those that might have been treated or laboratory grown, but IR analysis cannot determine the color origin of a diamond. Many types of detection equipment based upon other methods, such as Vis-NIR absorption spectroscopy or fluorescence spectroscopy, indirectly ascertain the diamond type. The assessment of such detection methods is beyond the scope of this article; however, third-party testing and evaluation of common laboratory-grown diamond detection equipment is available (e.g., https://www.naturaldiamonds.com/council/assuretesting-program//. More details regarding growth methods and identification criteria of CVD- and HPHT-grown diamonds with D-to-Z color have been previously documented (Wang et al., 2012; D'Haenens-Johansson et al., 2015; Eaton-Magaña and Shigley, 2016; Eaton-Magaña et al., 2017).

Figure 10. Graining in D-to-Z diamond is relatively common and related to displaced planes of carbon atoms in the atomic lattice (A). Brown graining indicates vacancy cluster defects along the areas of distortion (B), while reflective or interference (rainbow) graining is a product of light interaction with the grainlines (C and D). Even after HPHT treatment has removed the brown color to decolorize a diamond, remnant transparent graining can often still be seen (E). Photomicrographs by Kyaw Soe Moe (A), Vincent Cracco (B and C), and John I. Koivula (D and E).
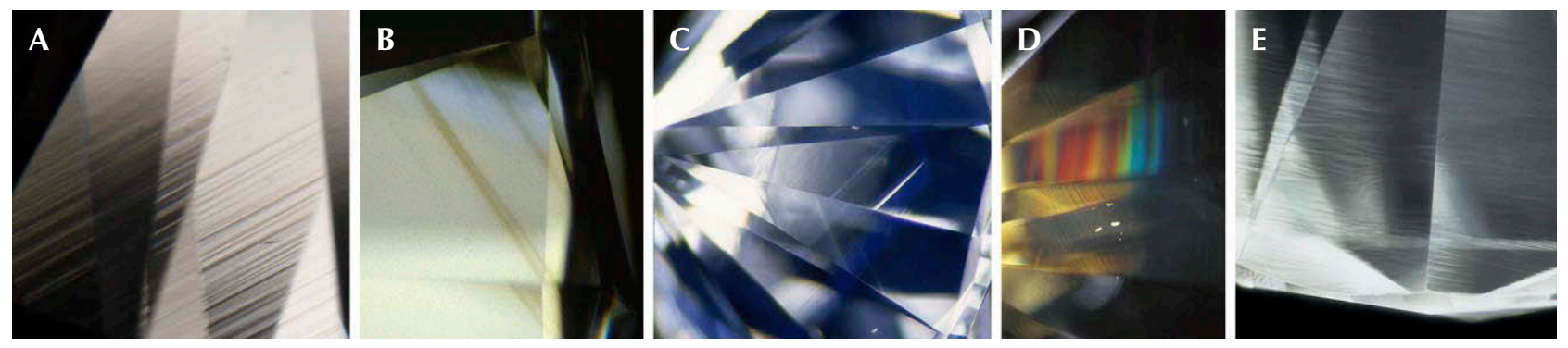


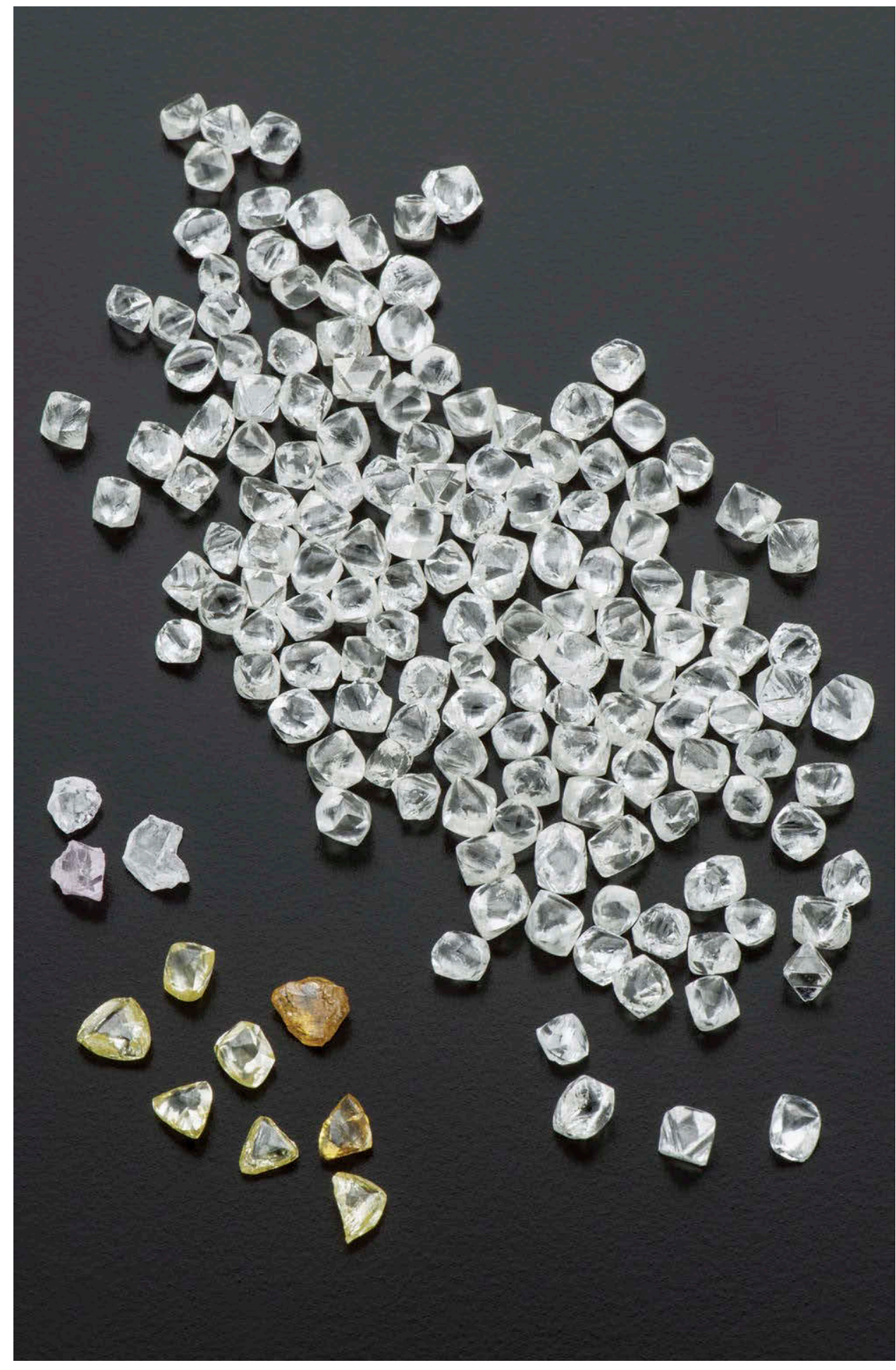

Figure 11. Colorless diamonds are a cornerstone of the gem trade. Photo by Robert Weldon.

\section{CONCLUSIONS}

Previous research has shown that most type I and type II diamonds (distinguished by the presence/absence of detectable nitrogen in their IR spectra) formed under very different conditions (Shirey and Shigley, 2013;
Smith et al., 2016, 2018). These differences extend to the faceted diamonds studied here. We examined the grading quality factors (among other properties) on all diamonds submitted to all GIA laboratories during much of 2017, and these showed quite distinct distri- 
butions. A majority of type II and type IaB diamonds show D color. For type II diamonds, the average carat weight is greater and the cut grades are higher. Additionally, type II diamonds show a bimodal clarity distribution, with a significant percentage of both type IIa and type IIb showing IF clarity (or VVS clarity that is perhaps improvable to IF), while the remaining type II diamonds more closely resemble the clarity distribution of type I.

In a separate examination, we looked at the distribution of grading quality factors for HPHT-treated diamond (box B). The vast majority of these are large type IIa diamonds, treated at a sufficiently high temperature to become colorless. Due to the adverse effects of HPHT treatment on lower-clarity diamonds, the majority of the stones chosen are in the VVS range.

Most people are familiar with colorless to nearcolorless diamonds and GIA's 4Cs grading system for evaluating them. However, analyzing the distribution across the grading range for such a large sample set illuminated some heretofore unseen trends. The majority of the world's diamonds that have been submitted to GIA - type Ia/IaAB that likely formed in the continental lithosphere-generally show high quantities of nitrogen and often have no observable fluorescence. Type II diamonds show some distinctly different patterns in grading quality factors that likely correspond with their unique formation, along with cutting decisions stemming from those differences.

Although this article compiles and combines data for an enormous dataset of the world's diamonds (figure 11), each stone remains unique. No two natural diamonds are alike. Each has its own specific clarity plot, IR spectrum, faceting pattern, and formation story.

\section{ABOUT THE AUTHORS}

Dr. Eaton-Magaña is a senior manager of diamond identification, Mr. Ardon is a research associate, Dr. Breeding is a senior manager of analytics, and Dr. Shigley is a distinguished research fellow, at GIA in Carlsbad, California.

\section{ACKNOWLEDGMENTS}

We thank Alex Balter for his instrumental help in retrieving the grading quality factor data for the diamonds in these datasets, as well as Jenny "Po Ting" Ng for reviewing images. Tom Moses, John King, Al Gilbertson, Dr. Wuyi Wang, Dr. Evan Smith, and Dr. Ulrika D'Haenens-Johansson read early versions of this manuscript and provided invaluable advice and perspective. We also thank the peer reviewers for their many comments and suggestions that improved the quality of the manuscript.

\section{REFERENCES}

Bouman M., Anthonis A., Chapman J., Smans S., De Corte K. (2018) The effect of blue fluorescence on the colour appearance of round-brilliant-cut diamonds. Journal of Gemmology, Vol. 36, No. 4, pp. 298-315.

Boyd S.R., Kiflawi I., Woods G.S. (1995) Infrared absorption by the B nitrogen aggregate in diamond. Philosophical Magazine B, Vol. 72, No. 3, pp. 351-361, http://dx.doi.org/10.1080/ 13642819508239089

Breeding C.M., Eaton-Magaña S.C. (2019) Fluorescence of natural and synthetic gem diamond: Mechanism and applications. Encyclopedia of Analytical Chemistry, John Wiley \& Sons, Ltd.

Breeding C.M., Shigley J.E. (2009) The "type" classification system of diamonds and its importance in gemology. $G \uplus G$, Vol. 45, No. 2, pp. 96-111, http://dx.doi.org/10.5741/GEMS.45.2.96

Breeding C.M., Eaton-Magaña S.C., Shigley J.E. (2018) Naturalcolor green diamonds: A beautiful conundrum. $G \uplus G$, Vol. 54, No. 1, pp. 2-27, http://dx.doi.org/10.5741/GEMS.54.1.2

Breeding C.M., Eaton-Magaña S.C., Shigley J.E. (2020) Naturally colored yellow and orange gem diamonds: The nitrogen factor. GÆG, Vol. 56, No. 2, pp. 194-219, http://dx.doi.org/10.5741/ GEMS.56.2.194

Caspi A. (1997) Modern diamond cutting and polishing. $G \uplus G$, Vol. 33, No. 2, pp. 103-121, http://dx.doi.org/10.5741/GEMS.33.2.102
Chalain J.P., Fritsch E., Hänni H.A. (1999) Detection of GEPOL diamonds, a first stage. Revue de. Gemmologie, No. 138-139, pp. 30-33.

Collins A.T. (1992) The characterisation of point defects in diamond by luminescence spectroscopy. Diamond and Related Materials, Vol. 1, No. 5/6, pp. 457-469, http://dx.doi.org/10.1016/09259635(92) $90146-\mathrm{F}$

Davies G., Crossfield M. (1973) Luminescence quenching and zerophonon line broadening associated with defect interactions in diamond. Journal of Physics C: Solid State Physics, Vol. 6, No. 5, L104-L108, http://dx.doi.org/10.1088/0022-3719/6/5/007

D'Haenens-Johansson U.F., Katrusha A., Johnson P., Wang W. (2015) Large colorless HPHT-grown synthetic gem diamonds from New Diamond Technology, Russia. Ge G, Vol. 51, No. 3, pp. 260-279, http://dx.doi.org/10.5741/GEMS.51.3.260

Dirlam D.M., Shigley J.E., Overlin S.D. (2002) The ultimate gemologist: A tribute to Richard T. Liddicoat. $G \uplus G$, Vol. 38, No. 1, pp. 2-13, http://dx.doi.org/10.5741/GEMS.38.1.2

Dobrinets I.A., Vins V.G., Zaitsev A.M. (2013) HPHT-Treated Diamonds: Diamonds Forever. Springer, Heidelberg.

Eaton-Magaña S.C., Shigley J.E. (2016) Observations on CVDgrown synthetic diamonds: A review. $G \uplus G$, Vol. 52, No. 3, pp. 222-245, http://dx.doi.org/10.5741/GEMS.52.3.222 
Eaton-Magaña S.C., Shigley J.E., Breeding C.M. (2017) Observations on HPHT-grown synthetic diamonds: A review. $G \uplus G$, Vol. 53, No. 3, pp. 262-284, http://dx.doi.org/10.5741/GEMS.53.3.262

Eaton-Magaña S.C., Breeding C.M., Shigley J.E. (2018a) Natural-color blue, gray, and violet diamonds: Allure of the deep. $G \uplus G$, Vol. 54, No. 2, pp. 112-131, http://dx.doi.org/10.5741/GEMS.54.2.112

Eaton-Magaña S.C., Ardon, T., Smit K.V., Breeding C.M., Shigley J.E. (2018b) Natural-color pink, purple, red, and brown diamonds: Band of many colors. GÆ G, Vol. 54, No. 4, pp. 352377, http://dx.doi.org/10.5741/GEMS.54.2.352

Eaton-Magaña S.C., Ardon T., Breeding C.M., Shigley J.E. (2019) Natural-color fancy white and fancy black diamonds: Where color and clarity converge. $G \uplus G$, Vol. 55, No. 3, pp. 320-327, http://dx.doi.org/10.5741/GEMS.55.3.320

Fang C., Zhang Y., Zhang Z., Shan C., Shen W., Jia X. (2018) Preparation of "natural" diamonds by HPHT annealing of synthetic diamonds. Crystal Engineering Communications, Vol. 20, No. 4, pp. 505-511, http://dx.doi.org/10.1039/C7CE02013A

Fisher D., Spits R.A. (2000) Spectroscopic evidence of GE POL HPHT-treated natural type IIa diamonds. Ge G, Vol. 36, No. 1, pp. 42-49, http://dx.doi.org/10.5741/GEMS.36.1.42

Fisher D., Sibley S.J., Kelly C.J. (2009) Brown colour in natural diamond and interaction between the brown related and other colour-inducing defects. Journal of Physics: Condensed Matter, Vol. 21, No. 36, Article 364213, 10 pp., http://dx.doi.org/10.1088/0953-8984/21/36/364213

Gaillou E., Post J.E., Bassim N.D., Zaitsev A.M., Rose T., Fries M.D., Stroud R.M., Steele A., Butler J.E. (2010) Spectroscopic and microscopic characterizations of color lamellae in natural pink diamonds. Diamond and Related Materials, Vol. 19, No. 10, pp. 1207-1220, http://dx.doi.org/10.1016/j.diamond.2010.06.015

Geurts R.H., Reinitz I.M., Blodgett T., Gilbertson A.M. (2011) GIA's symmetry grading boundaries for round brilliant cut diamonds. $G \uplus G$, Vol. 47, No. 4, pp. 286-295, http://dx.doi.org/10.5741/GEMS.47.4.286

Goss J.P., Briddon P.R., Hill V., Jones R., Rayson M.J. (2014) Identification of the structure of the $3107 \mathrm{~cm}^{-1} \mathrm{H}$-related defect in diamond. Journal of Physics: Condensed Matter, Vol. 26, No. 14, pp. 1-6, http://dx.doi.org/10.1088/0953-8984/26/14/145801

Gu T., Wang W. (2017) IaB diamond and its geological implications. International Kimberlite Conference: Extended Abstracts, Vol. 11

Hemphill T.S., Reinitz I.M., Johnson M.L., Shigley J.E. (1998) Modeling the appearance of the round brilliant cut diamond: An analysis of brilliance. $G \uplus G$, Vol. 34, No. 3, pp. 158-183, http://dx.doi.org/10.5741/GEMS.34.3.158

Hounsome L.S., Jones R., Martineau P.M., Fisher D., Shaw M.J., Briddon P.R., Oberg S. (2006) Origin of brown coloration in diamond. Physical Review B, Vol. 73, No. 12, Article 125203 , http://dx.doi.org/10.1103/PhysRevB.73.125203

Kagi H., Zedgenizov D.A., Ohfuji H., Ishibashi H. (2016) Microand nano-inclusions in a superdeep diamond from São Luiz, Brazil. Geochemistry International, Vol. 54, No. 10, pp. 834838, http://dx.doi.org/10.1134/S0016702916100062

Kaminsky F.V., Zakharchenko O.D., Griffin W.L., Channer D.M.D., Khachatryan-Blinova G.K. (2000) Diamond from the Guaniamo area, Venezuela. The Canadian Mineralogist, Vol. 38, No. 6, pp. 1347-1370, http://dx.doi.org/10.2113/gscanmin.38.6.1347

Kiflawi I., Mayer A.E., Spear P.M., Van Wyk J.A., Woods G.S. (1994) Infrared absorption by the single nitrogen and A defect centres in diamond. Philosophical Magazine B, Vol. 69, No. 6, pp. 1141-1147, http://dx.doi.org/10.1080/01418639408240184

King J.M., Moses T.M., Wang W. (2006) The impact of internal whitish and reflective graining on the clarity grading of $\mathrm{D}$-to- $\mathrm{Z}$ color diamonds at the GIA Laboratory. Ge G, Vol. 42, No. 4, pp. 206-220, http://dx.doi.org/10.5741/GEMS.42.4.206

King J.M., Geurts R.H., Gilbertson A.M., Shigley J.E. (2008) Color grading "D-to-Z" diamonds at the GIA Laboratory. Ge) G, Vol. 44, No. 4, pp. 296-321, http://dx.doi.org/10.5741/GEMS.44.4.296
Koivula J.I. (2000) The Microworld of Diamonds: A Visual Reference Guide. Gemworld International, Inc., Northbrook, IL, 157 pp.

Krawitz A. (2008) Graff unveils Lesotho Promise necklace. https://www.diamonds.net/news/NewsItem.aspx?ArticleID =2 2702 [Accessed: 2/19/2020]

Luo Y., Breeding C.M. (2013) Fluorescence produced by optical defects in diamond: Measurement, characterization, and challenges. $G \uplus G$ Vol. 49, No. 2, pp. 82-97, http://dx.doi.org/10.5741/GEMS.49.2.82

Moses T.M., Reinitz I.M., Johnson M.L., King J.M., Shigley J.E. (1997) A contribution to understanding the effect of blue fluorescence on the appearance of diamonds. $G \uplus G$, Vol. 33, No. 4 pp. 244-259, http://dx.doi.org/10.5741/GEMS.33.4.244

Moses T.M., Johnson M.L., Green B., Blodgett T., Cino K., Geurts R.H., Gilbertson A.M., Hemphill T.S., King J.M., Kornylak L., Reinitz I.M., Shigley J.E. (2004) A foundation for grading the overall cut quality of round brilliant cut diamonds. $G \oplus G$, Vol 40, No. 3, pp. 202-229, http://dx.doi.org/10.5741/GEMS.40.3.202

Renfro N.D., Koivula J.I., Muyal J., McClure S.F., Schumacher K., Shigley J.E. (2018) Inclusions in natural, synthetic, and treated diamond. $G \uplus G$, Vol. 54, No. 4, pp. 428-429, http://dx.doi.org/10.5741/GEMS.54.4.428

Robertson R., Fox J.J., Martin A.E. (1933) Two types of diamonds. Philosophical Transactions of the Royal Society A, Vol. 232, No 707-720, pp. 463-535, http://dx.doi.org/10.1098/rsta.1934.0013

Rudloff-Grund J., Brenker F.E., Marquardt K., Howell D., Schreiber A., O'Reilly S.Y., Griffin W.L., Kaminsky F.V. (2016) Nitrogen nanoinclusions in milky diamonds from Juina area, Mato Grosso State, Brazil. Lithos, Vol. 265, pp. 57-67, http://dx.doi.org/10.1016/i.lithos.2016.09.022

Shirey S.B., Shigley J.E. (2013) Recent advances in understanding the geology of diamonds. $G \uplus G$, Vol. 49, No. 4, pp. 188-222, http://dx.doi.org/10.5741/GEMS.49.4.188

Smit K.V., Shor R. (2017) Geology and development of the Lomonosov diamond deposit, northwestern Russia. $G \uplus G$, Vol. 53, No. 2, pp. 144-167, http://dx.doi.org/10.5741/GEMS.53.2.144

Smit K.V., Myagkaya E., Persaud S., Wang W. (2018) Black diamonds from Marange (Zimbabwe): A result of natural irradiation and graphite inclusions. Ge G, Vol. 54, No. 2, pp. 132-148, http://dx.doi.org/10.5741/GEMS.54.2.132

Smith E.M., Shirey S.B., Nestola F., Bullock E.S., Wang J., Richardson S.H., Wang W. (2016) Large gem diamonds from metallic liquid in Earth's deep mantle. Science, Vol. 354, No. 6318, pp. 1403-1405, http://dx.doi.org/10.1126/science.aal1303

Smith E.M., Shirey S.B., Wang W. (2017) The very deep origin of the world's biggest diamonds. GÆG, Vol. 53, No. 4, pp. 388403, http://dx.doi.org/10.5741/GEMS.53.4.388

Smith E.M., Shirey S.B., Richardson S.H., Nestola F., Bullock E.S., Wang J., Wang W. (2018) Blue boron-bearing diamonds from Earth's lower mantle. Nature, Vol. 560, No. 7716, pp. 84-87, http://dx.doi.org/10.1038/s41586-018-0334-5

Tang C.J., Neves A.J., Carmo M.C. (2005) On the two-phonon absorption of CVD diamond films. Diamond and Related Materials, Vol. 14, No. 11/12, pp. 1943-1949, http://dx.doi.org/ 10.1016/j.diamond.2005.08.060

Vasil'ev E.A., Ivanov-Omskii V.I., Pomazanskii B.S., Bogush I.N. (2004) The N3 center luminescence quenched by nitrogen impurity in natural diamond. Technical Physics Letters, Vol. 30, No. 10, pp. 802-803, http://dx.doi.org/10.1134/1.1813714

Wang W. (2010) Lab Notes: Lab sees increasing number of large HPHT-treated type IIa diamonds. Ge G, Vol. 46, No. 4, p. 298

Wang W., D'Haenens-Johansson U.F., Johnson P., Moe K.S., Emerson E., Newton M.E., Moses T.M. (2012) CVD synthetic diamonds from Gemesis Corp. G»G, Vol. 48, No. 2, pp. 80-97, http://dx.doi.org/10.5741/GEMS.48.2.80

Woods G.S. (1986) Platelets and the infrared absorption of type Ia diamonds. Proceedings of the Royal Society of London A, Vol. 407, No. 1832, pp. 219-238, http://dx.doi.org/10.1098/rspa.1986.0094

Zaitsev A.M. (2003) Optical Properties of Diamond: A Data Handbook. Springer, Berlin. 